\title{
Species- and C-terminal linker-dependent variations in the dynamic behavior of FtsZ on membranes in vitro
}

\author{
Kousik Sundararajan ${ }^{1}$, DR. Anthony Vecchiarelli ${ }^{2}$, Kiyoshi Mizuuchi ${ }^{3}$, and DR. Erin D. \\ Goley ${ }^{1, *}$ \\ ${ }^{1}$ Department of Biological Chemistry, Johns Hopkins University School of Medicine, Baltimore, \\ Maryland, 21205 \\ ${ }^{2}$ Molecular, Cellular, and Developmental Biology, University of Michigan College of Literature \\ Science and the Arts, Ann Arbor, Michigan, 48109 \\ ${ }^{3}$ Laboratory of Molecular Biology, National Institute of Diabetes, and Digestive and Kidney \\ Diseases, National Institutes of Health, Bethesda, Bethesda, Maryland 20814
}

\section{Summary:}

Bacterial cell division requires the assembly of FtsZ protofilaments into a dynamic structure called the 'Z-ring'. The Z-ring recruits the division machinery and directs local cell wall remodeling for constriction. The organization and dynamics of protofilaments within the Z-ring coordinate local cell wall synthesis during cell constriction, but their regulation is largely unknown. The disordered C-terminal linker (CTL) region of Caulobacter crescentus FtsZ ( $C$ cFtsZ) regulates polymer structure and turnover in solution in vitro, and regulates Z-ring structure and activity of cell wall enzymes in vivo. To investigate the contributions of the CTL to the polymerization properties of FtsZ on its physiological platform, the cell membrane, we reconstituted $C c$ FtsZ polymerization on supported lipid bilayers (SLB) and visualized polymer dynamics and structure using total internal reflection fluorescence microscopy. Unlike E. coli FtsZ protofilaments that organized into large, bundled patterns, $C c$ FtsZ protofilaments assembled into small, dynamic clusters on SLBs. Moreover, $C c$ FtsZ lacking its CTL formed large networks of straight filament bundles that underwent slower turnover than the dynamic clusters of wildtype FtsZ. Our in vitro characterization provides novel insights into species- and CTL-dependent differences between FtsZ assembly properties that are relevant to Z-ring assembly and function on membranes in vivo.

\section{Abbreviated Summary:}

The division machinery in bacteria assembles on a dynamic cytokinetic ring made of FtsZ filaments. Here, we reconstitute $C$. crescentus FtsZ on supported lipid bilayers and demonstrate using microscopy that $E$. coli and $C$. crescentus FtsZs have distinct emergent superstructures despite forming similar precursors. Additionally, we determine that the disordered C-terminal

"Corresponding author: egoley1@jhmi.edu. Author Contributions

$\mathrm{KS}, \mathrm{AV}, \mathrm{KM}$, and EDG designed the experiments. KS and AV performed the experiments. KS analyzed the data. KS, AV, KM, and EDG wrote the paper and approved the final version of the manuscript.

Conflict of Interest

The authors declare they have no conflict of interests with the contents of this manuscript. 
linker of $C$. crescentus FtsZ regulates the dynamic assembly of superstructures on membrane, which might be relevant for its regulation of cell wall metabolism.

\section{Abstract}

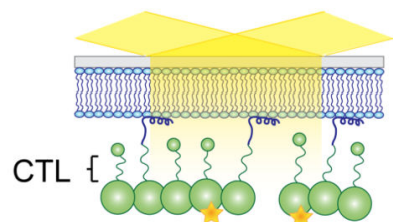

\section{TIRFM}

Supported lipid bilayer

FtsZ/FtsZ-MTS polymers
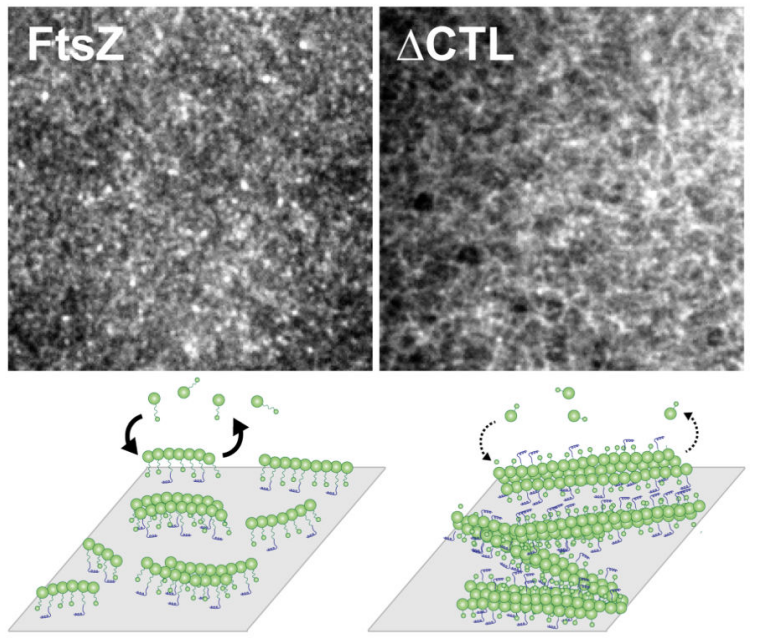

\section{Keywords}

FtsZ; Caulobacter crescentus; polymerization; cell division; in vitro reconstitution

\section{Introduction:}

In bacteria, the process of cytokinesis requires remodeling of the cell wall at the division site following the assembly of the multi-protein division complex termed the divisome. The tubulin homolog FtsZ polymerizes and forms a ring-like scaffold called the "Z-ring" at the incipient division site for the recruitment of the divisome. FtsZ protofilaments assemble into dynamic clusters that together form a discontinuous Z-ring (Li et al., 2007; Fu et al., 2010; Holden et al., 2014; Bisson-Filho et al., 2017; Yang et al., 2017). Following assembly of the Z-ring, more than two dozen factors are recruited to the division site through direct or indirect interactions with FtsZ (Erickson et al., 2010; Meier and Goley, 2014). Through the recruitment of cell wall enzymes, the Z-ring promotes local cell wall synthesis (Aaron et al., 2007). In addition to their recruitment, the Z-ring also regulates the activity of these enzymes at the site of division (Sundararajan et al., 2015). Recent studies of FtsZ have suggested that the dynamics of clusters of protofilaments in the Z-ring result in an apparent directional movement of clusters through treadmilling (Bisson-Filho et al., 2017; Yang et al., 2017). Moreover, the direction and speed of these clusters are correlated with the direction and speed of movement of cell wall enzymes required for cell division. Thus, it appears that the polymerization properties of FtsZ are essential for its function in local cell wall 
remodeling during cytokinesis (Sundararajan et al., 2015; Bisson-Filho et al., 2017; Yang et al., 2017). However, the regulation of the assembly of FtsZ into dynamic clusters, the higher-order arrangement of protofilaments within the clusters, and the source of directional dynamic assembly of these clusters are largely unknown.

In cells, FtsZ protofilaments observed by electron cryotomography appear as slightly curved protofilaments running circumferentially along the short axis of the cell near the inner membrane (Li et al., 2007; Szwedziak et al., 2014). In vitro, FtsZ polymerizes on binding GTP into single protofilaments, straight multifilament bundles, helical bundles or toroids depending on the presence of binding factors, crowding agents or divalent cations, as observed by electron microscopy (Mukherjee and Lutkenhaus, 1999; Gueiros-Filho and Losick, 2002; Popp et al., 2009; Goley et al., 2010). It is unclear which of the structures of FtsZ polymers observed in vitro are physiologically relevant in cells, especially in the context of attachment to the membrane. Efforts to observe dynamic assembly of FtsZ polymers on a membrane have been limited to E. coli FtsZ (Arumugam et al., 2012; Loose and Mitchison, 2014; Arumugam et al., 2014; Ramirez-Diaz et al., 2018). In particular, purified E. coli FtsZ assembles into large, dynamic bundles of treadmilling protofilaments when anchored to a membrane either using an artificial membrane targeting sequence (MTS) or membrane-anchoring proteins such as FtsA and observed by total internal reflection fluorescence microscopy (TIRFM) in vitro (Loose and Mitchison, 2014; Ramirez-Diaz et al., 2018). The conservation and physiological relevance of these emergent structures of FtsZ protofilaments on membranes have yet to be demonstrated.

FtsZ polymerizes through its conserved tubulin-like GTPase domain. The GTPase domain is followed by a C-terminal tail made of an intrinsically disordered region (C-terminal linker or CTL) and a conserved peptide region (C-terminal conserved peptide or CTC) for binding membrane-anchoring proteins of FtsZ (Vaughan et al., 2004; Erickson et al., 2010). In Caulobacter crescentus, E. coli and B. subtilis, FtsZ requires the CTL to assemble into a functional Z-ring capable of cytokinesis (Buske and Levin, 2013; Gardner et al., 2013; Sundararajan et al., 2015). In $C$. crescentus cells, expression of FtsZ lacking its CTL ( $\Delta$ CTL) has dominant lethal effects on cell wall metabolism leading to cell filamentation, local envelope bulging and rapid lysis (Sundararajan et al., 2015). Although $\Delta C T L$ is functional for recruiting all of the known FtsZ binding proteins and directing local cell wall synthesis, it causes defects in the chemistry of the cell wall that lead to cell lysis (Sundararajan et al., 2015). The CTL thus contributes to the ability of FtsZ to regulate cell wall metabolism, independent of FtsZ's function as a scaffold for localizing cell wall enzymes. The structures formed by $\triangle \mathrm{CTL}$ in cells appear deformed when compared to wildtype FtsZ - they are larger, brighter, and less ring-like by epifluorescence microscopy. In vitro, $\Delta \mathrm{CTL}$ polymerizes into long, straight multifilament bundles with low GTP hydrolysis rates compared to single slightly curved protofilaments of wildtype FtsZ (Sundararajan and Goley, 2017). Since the CTL appears to affect both FtsZ dynamics and polymer structure in vitro, it is still unclear if the CTL-dependent effects on cell wall metabolism are through the regulation of rates of cell wall synthesis, the regulation of higher order spatial organization of cell wall enzymes, and/or the coordination of the activities of different enzymes at the site of division. How the most variable region of FtsZ across organisms - the CTL - contributes 
to the higher order assembly of the Z-ring and its function in cell wall metabolism is not fully understood.

Here, we have developed an in vitro TIRFM-based assay to image FtsZ polymers anchored to supported lipid bilayers (SLB) through an artificial membrane tethering sequence peptide (MTS) from E. coli MinD (Osawa et al., 2008). Unlike prior reconstitution studies of FtsZ polymerization on membrane constrained within wells placed on coverslips (Loose and Mitchison, 2014; Arumugam et al., 2014; Ramirez-Diaz et al., 2018), we adapted a system to allow for rapid depletion and repletion experiments in a controlled environment using flow cells (Vecchiarelli et al., 2016). Whereas the well set-up employs series of dilutions for the removal of components (protein, nucleotide etc.) from the reaction chamber, the use of microfluidics in our flowcell setup enables us to observe FtsZ polymer behavior on membranes during changes in reaction conditions, in addition to observing polymer dynamics and structure at steady state. Using our flow cell setup, we observe that whereas $E$. coli FtsZ assembles into large, dynamic bundles as shown previously (Loose and Mitchison, 2014; Arumugam et al., 2014; Ramirez-Diaz et al., 2018), C. crescentus FtsZ assembles into smaller dynamic clusters under identical in vitro conditions. Investigating the effects of the CTL on FtsZ polymerization, we observe that $\triangle \mathrm{CTL}$ forms large networks of straight filaments on SLBs that turn over more slowly compared to the dynamic clusters formed by WT FtsZ. We conclude that the CTL is required for disrupting lateral interaction between protofilaments and promoting polymer turnover on membranes. Our study provides the first in vitro characterization of assembly and dynamics on SLBs of FtsZ from an organism other than E. coli and describes CTL-dependent regulation of FtsZ polymerization that we propose is relevant to FtsZ-mediated regulation of cell wall metabolism in cells.

\section{Results}

\section{C. crescentus FtsZ assembles into dynamic superstructures on supported lipid bilayers}

To image FtsZ polymer assembly on SLBs, we adapted the flow cell setup developed for observing MinD-MinE protein oscillations on membranes (Vecchiarelli et al., 2016). Specifically, we coated flow cells with SLBs composed of combinations of synthetic anionic (DOPG) and zwitterionic (DOPC) lipids (Figure 1A). To visualize FtsZ filaments anchored to the membrane, we used fluorescently labeled FtsZ fused to the membrane targeting sequence (MTS) from E. coli MinD, or a mixture of unlabeled (non-fluorescent) FtsZ fused to MTS and fluorescently labeled FtsZ that had no MTS to generate copolymers. FtsZ variants were incubated with GTP and flowed into the SLB-coated flow cell $(\sim 3 \mu \mathrm{L}$ total volume). FtsZ polymers on the membrane were then imaged using prism-type total internal reflection fluorescence microscopy (TIRFM) (Figure 1A).

To compare our reconstitution approach to previously published studies on FtsZ polymers on SLBs, we first examined structures formed by $E$. coli FtsZ with the YFP derivative venus and MTS fused to its C-terminus in tandem and replacing the CTC $\left(E c\right.$ His $_{6}$-FtsZ-venusMTS) (Osawa et al., 2008). This is modeled after Ec FtsZ-YFP-MTS which has been used in the past to observe E. coli FtsZ polymerization on membranes, within vesicles as well as on planar SLB (Osawa et al., 2008; Osawa et al., 2009; Osawa and Erickson, 2011; Arumugam et al., 2012; Osawa and Erickson, 2013; Loose and Mitchison, 2014; Arumugam et al., 2014; 
Ramirez-Diaz et al., 2018). When we flowed in $2 \mu \mathrm{M} E c$ His $_{6}$-FtsZ-venus-MTS premixed with $2 \mathrm{mM}$ GTP for 30 minutes, we observed dynamic assembly of fluorescent clusters on the membrane (Figure 1B, Movie 1.1). The clusters were typically amorphous or circular, measuring $413 \mathrm{~nm} \pm 53 \mathrm{~nm}$ along short axis and $615 \mathrm{~nm} \pm 194 \mathrm{~nm}$ along long axis (mean \pm S.D., $\mathrm{n}=28$ ) at the start of imaging (Figure 1C, 1D). These measurements are likely overestimations of the actual dimensions of the structures due to the resolution-limit of light microscopy $(\sim 250 \mathrm{~nm})$. Within 5 minutes of assembly, the amorphous clusters extended into dynamic filaments with variable lengths $(980 \pm 350 \mathrm{~nm}$, mean \pm S.D., $\mathrm{n}=29)$ and relatively tighter distribution of widths ( $381 \mathrm{~nm} \pm 59 \mathrm{~nm}$, mean \pm S.D., $\mathrm{n}=29)$ (Figure 1B, 1C, 1D). On further incubation, these filaments aligned to form parallel filament bundles with periodic fluorescence intensity fluctuations along the length of the bundles (Figure 1D, 1E, Movie 1.1). The widths of filament bundles (full width half maximum distances of the fluorescence intensity plot along the short axis) were typically comparable to the widths of their precursors ( $425 \mathrm{~nm} \pm 84 \mathrm{~nm}$, mean \pm S.D., $\mathrm{n}=25$ ) (Figure 1D). The bundles that constituted these structures appear similar in dimensions and dynamics to published observations of assembly on SLB-coated wells of Ec FtsZ-YFP-MTS (Arumugam et al., 2014; Ramirez-Diaz et al., 2018) or Ec FtsZ polymers with the membrane-anchoring protein ZipA (Loose and Mitchison, 2014).

When we flowed $1.8 \mu \mathrm{M} C$. crescentus FtsZ-venus-MTS preincubated with $2 \mathrm{mM}$ GTP for 30 minutes into the flow cell, we observed the formation of amorphous or circular clusters (minimum width of $397 \mathrm{~nm} \pm 62 \mathrm{~nm}$, mean \pm S.D., $\mathrm{n}=26$ ) similar to those observed at early time points with $E c$ His $_{6}$-FtsZ-venus-MTS (Figure 1D, 1F, Movie 1.2). These clusters assembled into speckled patterns at steady state (Figure 1G, Movie 1.3) while the dimensions of speckles remained similar to their precursors (minimum width of $446 \mathrm{~nm}$ $\pm 77 \mathrm{~nm}$, mean \pm S.D., $\mathrm{n}=26$ ) (Figure 1D). It should be noted that due to crowding of fluorescent clusters at the membrane (particularly for $C c$ FtsZ-venus-MTS), the measurement of widths at steady state only represent the dimensions of the brightest spots. Whereas $E c$ His $_{6}$-FtsZ-venus-MTS filament bundles assembled into dynamic patterns, with the patterns themselves remaining stable for minutes (Figure 1E), the $C c$ FtsZ-venus-MTS protofilaments formed highly dynamic and irregular speckled patterns on the SLB surface (Figure $1 \mathrm{H}$ ). We conclude that $C$. crescentus FtsZ assembles dynamically on SLBs into superstructures that are distinct from the filamentous superstructures formed by E. coli FtsZ.

The presence of the $\mathrm{His}_{6}$ tag on FtsZ has been reported to affect lateral interaction between FtsZ protofilaments and hence, polymer structure (Redick et al., 2005, Olivia et al., 2003). We tested if the differences in superstructures of $E c$ His $_{6}$-FtsZ-venus-MTS and $C c$ FtsZvenus-MTS were the result of the effects of the $\mathrm{His}_{6}$ tag by imaging the structures formed by $E c$ FtsZ-venus-MTS. When we flowed in 1 or $2 \mu \mathrm{M} E c$ FtsZ-venus-MTS incubated with GTP, we observed dynamic clusters that elongated into filament bundles that aligned into regular patterns on the SLB (Movie 1.4, 1.6, Supplementary figure 1A, 1C) similar to those observed for $E_{c} \mathrm{His}_{6}$-FtsZ-venus-MTS. On the other hand, 1 or $2 \mu \mathrm{M} C c$ FtsZ-venus-MTS formed dynamic speckled pattern on the SLB (Movie 1.5, 1.7, Supplementary figure 1B, 1D). 
A recent characterization of $E c$ FtsZ-YFP-MTS on SLBs reported that the superstructures formed by $E c$ FtsZ depends on the surface concentration of FtsZ at the membrane (Ramirez-2018). We tested if the differences between the superstructures formed by $E c$ FtsZ-venus-MTS and $C c$ FtsZ-venus-MTS are due to differences in surface concentrations despite similar solution concentration of monomers. Hence, we tested if $C c$ FtsZ-venusMTS could assemble into bundled patterns similar to those observed for $E c$ FtsZ-venusMTS at similar surface concentrations. We observed that gradual inflow of $2 \mu \mathrm{M}$ (total concentration) FtsZ-venus-MTS into flow cells resulted in increasing average fluorescence intensity by TIRFM, consistent with an increase in surface concentration of fluorescent FtsZ polymers (Supplementary Figure 1E). When we flowed Ec FtsZ-venus-MTS polymers, we observed a gradual increase in fluorescence intensity (Supplementary Figure 1E).

Simultaneously, we observed the gradual appearance of dynamic clusters that transformed into elongating dynamic bundles, eventually assembling into dynamic filamentous patterns (Movie 1.6, Supplementary figure 1C). Further inflow of polymers resulted in overlapping bundled superstructures that covered the entire membrane. $C c$ FtsZ-venus-MTS polymers initially formed speckled structures (Movie 1.7, Supplementary figure 1D). Moreover, the increase in fluorescence intensity was delayed compared to $E c$ FtsZ-venus-MTS after flowing similar volume (Supplementary figure 1C, 1D, 1E). In contrast to the gradual metamorphosis of $E c$ FtsZ-venus-MTS superstructures from clusters to bundles and finally to filamentaous patterns, $C c$ FtsZ-venus-MTS formed fluorescent clusters at low average intensities and irregular speckled superstructures at higher average intensities. At the highest fluorescence intensities, $E c$ FtsZ and $C c$ FtsZ superstructures appear to completely saturate the membrane (Movie 1.6, 1.7, Supplementary figure 1C, 1D). Thus, despite comparable surface concentrations (as inferred from fluorescence intensity), $E c$ and $C c$ FtsZ polymers form different superstructures on the membrane. We conclude that the differences in the superstructures formed by $E c$ FtsZ and $C c$ FtsZ polymers on SLBs are not merely due to differences in surface concentrations.

\section{In addition to small dynamic clusters, $\mathrm{C}$. crescentus $\Delta \mathrm{CTL}$ forms very large multi-filament bundles on SLB}

To address the contributions of the CTL to the assembly properties of $C$. crescentus FtsZ on membranes, we compared FtsZ with $\triangle \mathrm{CTL}$ and other CTL variants. Since we are interested in the contributions of the unstructured C-terminal region of FtsZ, we decided against using the bulky fluorescent fusion (venus-MTS) at the C-terminus of our CTL variants. Instead, we used FtsZ or $\triangle \mathrm{CTL}$ fluorescently labeled with Alexa488 dye conjugated at the only cysteine residue in $C$. crescentus FtsZ (Cys123) to visualize polymers. In addition to FtsZ or $\triangle C T L$ (a fraction of which was Alexa488-labeled), we also included equimolar unlabeled MTS-fusions of FtsZ or $\triangle \mathrm{CTL}$, wherein the CTC was replaced by the MTS, to recruit polymers to the membrane. To reduce variation due to slight differences in labeling efficiencies and to avoid increased background fluorescence due to crowding of fluorescent polymers at the membrane, we used unlabeled FtsZ- or $\triangle$ CTL-MTS (i.e. without Alexalabel) to recruit Alexa-labeled FtsZ or $\Delta \mathrm{CTL}$ to the membrane, respectively.

First, we confirmed that FtsZ-MTS could be used to specifically recruit FtsZ polymers to the membrane using a 1:1 mixture of FtsZ and FtsZ-MTS (Figure 2A). On introducing $2 \mu \mathrm{M}$ 
FtsZ (35\% FtsZ-Alexa488) pre-incubated with $2 \mathrm{mM} \mathrm{GTP} \mathrm{for} 5$ minutes into a flow cell equilibrated with $2 \mu \mathrm{M}$ FtsZ (35\% FtsZ-Alexa488), we observed a minor increase in fluorescence intensity above background levels (Figure 2B, 2D, Supplementary figure 2A, 2B, Movie 2.1). This increase was accompanied by the appearance of dynamic fluorescent clusters (Figure 2B, 2D, Supplementary figure 2A, 2B, Movie 2.1) suggesting the formation of FtsZ polymers in the solution phase that can transiently and assemble on or near the SLB surface. Strikingly, when we subsequently flowed in $2 \mu \mathrm{M}$ FtsZ (35\% FtsZ-Alexa488) and 2 $\mu \mathrm{M}$ FtsZ-MTS (unlabeled) pre-incubated with $2 \mathrm{mM}$ GTP, we observed a rapid increase in the number and intensity of fluorescent clusters (Figure 2C, Movie 2.2, Supplementary figure 2B). Since FtsZ-MTS is not fluorescently labeled, we conclude that the increase in intensity is due to the co-polymerization of FtsZ and FtsZ-MTS at the membrane bringing Alexa488-labeled FtsZ into the TIRF illumination field. At steady state, the dynamic clusters organized into speckled cloud-like patterns with fluctuating local fluorescence intensities (Movie 2.3, Supplementary figure 2C). Particularly, while we observed fluctuations in fluorescence intensity of the clusters in the order of seconds, their overall superstructure appeared to be maintained more stably over larger time periods (Supplementary figure 2C). On photobleaching, the FtsZ/FtsZ-MTS structures took $23.7 \mathrm{~s} \pm 1.9 \mathrm{~s}$ (mean \pm S.D., $\mathrm{n}=3$ ) to recover half the maximum intensity (Supplementary figure 2D), confirming that these structures are undergoing rapid turnover.

Next, we turned our attention to the role of the CTL in regulating FtsZ assembly on SLB. Since we could not attain labeling efficiency greater than $6 \%$ for $\triangle \mathrm{CTL}$, we used $6 \%$ Alexa488 labeled FtsZ or $\triangle \mathrm{CTL}$ in our experiments. On introduction of $2 \mathrm{mM}$ GTP into a reaction containing $2 \mu \mathrm{M}$ FtsZ (6\% Alexa488 labeled) with $2 \mu \mathrm{M}$ FtsZ-MTS, we observed structures similar to those observed for $2 \mu \mathrm{M}$ FtsZ (35\% Alexa488 labeled) with $2 \mu \mathrm{M}$ FtsZMTS (Figure 3A, Movie 3.1). By measuring change in intensity following the introduction of FtsZ-MTS or GTP into flow cells equilibrated with FtsZ and GTP, or FtsZ and FtsZ-MTS, correspondingly, we confirmed that the assembly of these structures on the SLB was MTSand GTP- dependent (Supplementary figure 3A, 3B).

Intriguingly, with $2 \mu \mathrm{M} \Delta \mathrm{CTL}$ (6\% Alexa488 labeled) and $2 \mu \mathrm{M} \Delta \mathrm{CTL}-\mathrm{MTS}$, we observed the rapid appearance of extended bright structures on the SLB following the introduction of GTP in addition to dynamic clusters similar to those observed for FtsZ/FtsZ-MTS (Figure 3A, 3B, Movie 3.2). Most of these structures oriented parallel to the direction of flow. After their rapid appearance, these structures underwent gradual decrease in fluorescence intensity, eventually dropping to background levels (Figure 3B, Supplementary figure 3C). At steady state, $\triangle \mathrm{CTL} / \Delta \mathrm{CTL}-\mathrm{MTS}$ protofilaments assembled as structures similar to FtsZ/ FtsZ-MTS (Figure 3A, Supplementary figure 3D). However, these structures were comparatively sparse - whereas the local intensities of FtsZ/FtsZ-MTS patterns appear diffuse when averaged over time, $\Delta$ CTL/ $\Delta$ CTL-MTS patterns contain more gaps between regions of high average intensities (Supplementary figure 3D). This result is in line with the sparse appearance of $\Delta \mathrm{CTL}$ polymers on electron microscopy grids and the lower steady state light scatter observed for $\triangle$ CTL compared to WT FtsZ in solution (Sundararajan et al 2015, Sundararajan and Goley 2017). 
The dimensions of the elongated structures of $\Delta \mathrm{CTL} / \Delta \mathrm{CTL}-\mathrm{MTS}$ on SLB are similar to the largest multi-filament bundles previously observed for $\Delta \mathrm{CTL}$ polymers by electron microscopy (Sundararajan and Goley, 2017). Such bundles were never observed for WT FtsZ or CTL variants, namely L14 (FtsZ with a 14 amino acid CTL) and HnCTL (FtsZ with CTL sequence from Hyphomonas neptunium) (Sundararajan and Goley, 2017). When we tested the assembly of L14/L14-MTS or $H n C T L / H n C T L-M T S$ copolymers on SLBs, we did not observe any elongated structures. Similar to FtsZ/FtsZ-MTS assembly on membranes, L14/L14-MTS and $H n C T L / H n C T L-M T S$ assembled into speckled cloud-like structures composed of dynamic fluorescent clusters at steady state (Figure 3A, Movie 3.3, Movie 3.4). The widths of the fluorescent clusters at steady state were not significantly different between the CTL variants $(\sim 700 \mathrm{~nm})$ (Supplementary figure 3E), however, this could be an overestimation due to the limit of resolution $(\sim 250 \mathrm{~nm})$ and low signal-to-noise ratio at steady state. From these observations, we conclude that the elongated structures observed specifically for $\Delta \mathrm{CTL} / \triangle \mathrm{CTL}-\mathrm{MTS}$ on SLB are large multi-filament bundles.

\section{In situ assembly/disassembly of FtsZ protofilaments on SLBS}

While the appearance of large $\Delta$ CTL/ $\Delta$ CTL-MTS bundles on SLBs is consistent with previous observations from electron microscopy that the CTL regulates lateral interaction between protofilaments (Sundararajan and Goley, 2017), we suspected that these structures are assembled in solution (upstream of the flow cell) during pre-incubation with GTP. Moreover, these structures are only observed during initial assembly of $\Delta \mathrm{CTL} / \Delta \mathrm{CTL}-\mathrm{MTS}$ polymers and are not observed at steady state. This suggests that these large, elongated bundles may only be relevant in solution and/or prior to their recruitment to the membrane. Because we are interested in observing the behavior of structures that form on membranes de novo, we therefore altered the flow cell setup to rapidly control the availability of GTP within the flow cell allowing us to induce polymerization (or depolymerization) in situ. We flowed the protein mixture and GTP through two separate, parallel inputs into the flow cell with equal flow rates (Figure 4A). During flow, the protein and GTP channels meet within the flow cell and maintain a laminar boundary (Figure 4A). As long as flow is maintained, the laminar boundary acts as a diffusion barrier and constrains polymerization to the interface between the protein and GTP channels (Figure 4B-E, Supplementary Figure 4A-D, Movies $4.1-4.4)$. When flow is stopped, the two channels mix by diffusion, rapidly initiating polymerization on the protein side due to the much faster diffusion of GTP compared to FtsZ monomers or polymers. Restarting flow rapidly depletes GTP from the protein side, thereby favoring depolymerization and disassembly of FtsZ polymers. Thus, by controlling the flow, we can initiate assembly and disassembly of FtsZ polymers in situ within the flow cell (Figure 4A).

To confirm that we can achieve such flow-dependent control on FtsZ assembly, we imaged the microfluidic chamber at 10× magnification while simultaneously flowing $2 \mu \mathrm{M}$ FtsZ (6\% FtsZ-Alexa488) and $2 \mu \mathrm{M}$ FtsZ-MTS mixture in the protein channel and $2 \mathrm{mM} \mathrm{GTP}$ in the GTP channel and subsequently stopping flow. Initially, on starting flow, we observed a rapid increase in fluorescence intensity only at the laminar boundary between the protein and GTP channels (Figure 4B, Supplementary Figure 4A, Movie 4.1). On the protein side, we observed a minor increase in fluorescence intensity likely due to unbound fluorescently 
labeled FtsZ monomers within the evanescent volume close to the SLB surface. On the GTP side, there was no significant increase in fluorescence intensity above background levels (Figure 4B, Supplementary Figure 4A, Movie 4.1). Immediately after the flow was stopped, we observed an increase in fluorescence intensity that spread gradually into the protein side, perpendicular to the original laminar boundary. On restarting flow, the average fluorescence intensity on the protein side decreased quickly until reaching levels comparable to background (Figure 4C, Supplementary Figure 4B, Movie 4.2). Subsequently, after the flow was stopped, the average fluorescence intensity increased once again, returning to values comparable to those observed before the flow was re-started (Figure 4C). The flowdependent changes in fluorescence intensities are as expected for diffusion-limited introduction (flow, then stop), depletion (subsequent flow) and repletion (subsequent stop) of GTP in the protein side, and the corresponding induction of polymerization, depolymerization, and repolymerization of FtsZ/FtsZ-MTS copolymers on the SLB (Figure 4A).

The fluorescence intensity profiles over time were comparable between flow cells with FtsZ/ FtsZ-MTS or $\Delta$ CTL/ $\Delta$ CTL-MTS (Figures 4B - 4E, Supplementary Figure 4A-D), with two major differences. Firstly, at steady state (no flow), flow cells with FtsZ/FtsZ-MTS attained higher local fluorescence intensity values on the protein side and lower local fluorescence intensity values at the original laminar boundary compared to $\Delta \mathrm{CTL} / \Delta \mathrm{CTL}-\mathrm{MTS}$ (Figure $4 \mathrm{~F}$, Supplementary Figure 4A-D, Supplementary figure 5A). Secondly, fluorescence intensity in flow cells with $\Delta \mathrm{CTL} / \Delta \mathrm{CTL}-\mathrm{MTS}$ took significantly longer to drop back to background levels on restarting flow as discussed below (Supplementary figure 5B). We also observed the appearance of many fluorescent puncta (spotty regions of high fluorescence intensity) on the SLBs on the protein side specifically with $\Delta$ CTL/ $\Delta$ CTL-MTS (Movie 4.3) and not with FtsZ/FtsZ-MTS (Movie 4.1). These puncta could be the result of aggregation or bundling of $\Delta \mathrm{CTL} / \Delta \mathrm{CTL}-\mathrm{MTS}$ protofilaments. These differences between FtsZ and $\Delta \mathrm{CTL}$ intensity profiles observed at 10× magnification suggest that the CTL influences higher order assembly of FtsZ polymers on membrane.

\section{$\triangle$ CTL polymers assemble into relatively stable filament networks on SLB}

Next, we observed the structures formed by FtsZ/FtsZ-MTS on the protein side of the original laminar boundary at 100× magnification. Immediately after stopping flow, we observed dynamic fluorescent clusters that assembled into speckled structures at steady state (Figure 5A, Figure 5B, Movie 5.1, 5.2) similar to our observations in the one-inlet flow cell setup. On restarting flow, these patterns gradually disassembled into sparse dynamic clusters that eventually disappear (Supplementary figure 5C, Movie 5.3). On stopping flow again, dynamic clusters reappear and form patterns distinct from those formed previously (before flow) (Supplementary figure 5C).

Strikingly, in addition to forming small dynamic clusters similar to those formed by FtsZ/ FtsZ-MTS, $\triangle \mathrm{CTL} / \Delta \mathrm{CTL}-\mathrm{MTS}$ structures formed elongated filament bundles that interconnected into a stable network (Figure 5B, Movie 5.4, 5.5). While the fluorescence intensities within the network showed rapid fluctuations, the $\Delta$ CTL/ $\Delta$ CTL-MTS network itself appeared stable for minutes (Figure 5C, Movie 5.4). These structures showed the 
highest fluorescence intensities in regions closest to the original laminar boundary on the protein channel side (Figure 4F). At regions of comparable surface concentrations (average fluorescence intensity), FtsZ/FtsZ-MTS did not form such networks of bundles and instead formed speckled patterns of dynamic clusters. This suggests that the distinct superstructures formed by $\Delta$ CTL/ $\triangle$ CTL-MTS polymers are not due to differences in the concentration of polymers on the membrane or in solution.

During initial flow of $\Delta \mathrm{CTL} / \Delta \mathrm{CTL}-\mathrm{MTS}$ in the protein channel, we observed large amorphous fluorescent clusters on the SLB on the protein side that rapidly appeared and gradually reduced in intensity until reaching background levels (Movie 5.4, Supplementary figure 5D). We could also observe such large fluorescent clusters at $10 \times$ magnification (Movie 4.3). These large clusters assemble on the SLB in a flow-dependent manner prior to the introduction of GTP (by stopping flow). The gradual disappearance of these clusters suggest that these are non-dynamic polymers or aggregates of $\Delta \mathrm{CTL} / \Delta \mathrm{CTL}-\mathrm{MTS}$ that are formed in solution during flow (Supplementary figure 5D). Unlike the 1-inlet setup, we did not observe long, thick, individual bundles of $\Delta$ CTL/ $\triangle$ CTL-MTS copolymers on the SLB when polymerized in situ.

In addition to the structural differences between the polymers formed by FtsZ/FtsZ-MTS and $\triangle \mathrm{CTL} / \triangle \mathrm{CTL}$-MTS on SLB, we also observed significant differences in their dynamics. When we rapidly depleted GTP from the protein side by restarting flow, we observed that the FtsZ structures disassembled at the rate of $3.6 \pm 0.1 \mathrm{~min}^{-1}$ (mean \pm S.D., $\mathrm{n}=4,2 \mu \mathrm{M}$ total protein), while $\Delta$ CTL/ $\Delta$ CTL-MTS structures disassembled at a slower rate of $2.1 \pm 0.5$ $\min ^{-1}$ (mean \pm S.D., $\mathrm{n}=3,2 \mu \mathrm{M}$ total protein) (Figure 5D, Supplementary figure 5E, 5F). The rate of disassembly of $\Delta \mathrm{CTL} / \Delta \mathrm{CTL}-\mathrm{MTS}$ structures were not significantly different at regions where the large fluorescent clusters or aggregates initially assembled prior to stoppage of flow. The decreased rate of disassembly of $\Delta$ CTL/ $\Delta$ CTL-MTS on depleting GTP mirrored the decreased rate of fluorescence recovery after photobleaching observed for these structures compared to FtsZ/FtsZ-MTS (Figure 5E). Whereas FtsZ/FtsZ-MTS took 13 $\mathrm{s} \pm 3 \mathrm{~s}$ (mean \pm S.D., $\mathrm{n}=3$ ) to recover $50 \%$ of fluorescence following photobleaching, $\Delta$ CTL/ $\Delta$ CTL-MTS took $42 \mathrm{~s} \pm 18 \mathrm{~s}$ (mean \pm S.D., $\mathrm{n}=3$ ) to recover fluorescence intensity with about 35\% loss in fluorescence intensity following photobleaching. These results indicate that the structures formed by $\Delta \mathrm{CTL} / \Delta \mathrm{CTL}$-MTS are more stable and have slower turnover compared to FtsZ/FtsZ-MTS.

\section{Discussion}

For polymerizing proteins such as FtsZ, their assembly properties are essential for their function. Observing the assembly of FtsZ polymerization in its physiological context is challenging in part due to the limitations of the spatio-temporal resolution of light microscopy and the complexity of multiple interacting components. In vitro reconstitution techniques have proven valuable for observing the assembly of dynamic cytoskeletal protein polymers from eukaryotes, and more recently, from bacteria. Electron microscopy has been used extensively for imaging FtsZ polymers, however its use is limited for observing polymer dynamics. On the other hand, GTP hydrolysis rate measurements and spectrofluorometric assays such as light scattering have been crucial for bulk measurements 
FtsZ polymerization in solution. However, these techniques provide little to no information on polymer structure. In the current study, we have described an in vitro reconstitution approach for observing FtsZ polymerization on planar SLBs, which provides both spatial and temporal resolution of FtsZ polymerization simultaneously. Moreover, it enables precise and rapid control of reaction conditions for observing the assembly and disassembly of FtsZ polymers, even during changes in reaction conditions, in addition to their steady state behavior.

As a validation of our approach, we demonstrate the reconstitution of $E c$ FtsZ-venus-MTS polymers into dynamic patterns (Figure 1B, Supplementary figures 1A, 1C) that are in agreement with the results of prior reconstitution efforts using E. coli FtsZ on SLBs (Arumugam et al., 2012; Loose and Mitchison, 2014; Arumugam et al., 2014; Ramirez-Diaz et al., 2018). Unlike for E. coli FtsZ or Ec FtsZ-venus-MTS on SLB, we never observed large-scale filamentous patterns formed by dynamic filament bundles for $C$. crescentus FtsZ on SLBs. Instead, we observed speckled patterns made of disconnected puncta or small dynamic clusters that move around in apparently random tracks (Figure 1F). Using our approach to understand the effects of the CTL on regulating lateral interaction between $C$. crescentus FtsZ protofilaments, we observed that $C$. crescentus $\Delta$ CTL forms networks of straight filamentous structures (Figure 5A, B) similar in scale to the multi-filament bundles observed by electron microscopy (Sundararajan and Goley, 2017). Moreover, we observed significantly slower dynamics for the higher order assembly of $\Delta$ CTL protofilaments compared to FtsZ protofilaments (Figure 5D, 5E). Thus, our approach provides valuable insights into $C$. crescentus FtsZ polymerization in the context of the membrane and complements the previous biochemical characterization of the effects of the CTL.

Interestingly, the precursors to the superstructures formed by $E c$ FtsZ and $C c$ FtsZ look comparable (Figure 1E). In both cases, dynamic clusters that are approximately $400 \mathrm{~nm}$ in diameter (or width) appear on the SLBs at the initial stage of polymer assembly. Similar dynamic clusters were observed with the CTL-variants of $C c$ FtsZ examined here, soon after the addition of GTP (Figure 3A). While the spatial resolution of the imaging system used here does not yield information on the organization of individual polymers within these nucleotide-dependent clusters, these clusters likely correspond to short individual protofilaments of FtsZ or bundles of a small number of short filaments, as observed by electron microscopy (Sundararajan and Goley, 2017). The assembly of $E c$ FtsZ and $C c$ FtsZ polymers into distinct dynamic superstructures at steady state despite the apparent similarity in their protofilament precursors is intriguing. In the case of $E c$ FtsZ, superstructures on SLBs are dependent on surface concentration of polymers (Ramirez-Diaz et al., 2018). The surface concentration is, in turn, dependent on the concentrations of GTP, free magnesium and/or membrane anchoring proteins such as FtsA (Loose and Mitchison, 2014; RamirezDiaz et al., 2018). In our flowcell setup, we observe that $E c$ FtsZ forms regular patterns of dynamic bundles at a wide range of surface concentrations and that $C c$ FtsZ does not form similar patterns at any surface concentration (Supplementary figure 1C, 1D, 1E). These observations suggest that differences in surface concentration alone cannot explain the differences between the superstructures formed by $E c$ FtsZ and $C c$ FtsZ. While the origin of these differences remain unclear, it is possible that a combination of variations in longitudinal and/or lateral interactions, average filament length, curvature, turnover, and/or 
treadmilling rates could result in $E c$ and $C c$ protofilaments assembly into distinct superstructures on membranes.

Which, if any, of the superstructures formed by $E c$ FtsZ or $C c$ FtsZ on SLBs in vitro are relevant in the physiological context of Z-ring assembly? Individual clusters of FtsZ protofilaments in Z-rings in vivo are asymmetric and shorter than $200 \mathrm{~nm}$ in length as observed by electron cryotomography (Li et al., 2007) or super-resolution light microscopy (Fu et al., 2010; Holden et al., 2014; Yang et al., 2017), most similar to the precursors of $C c$ FtsZ or $E c$ FtsZ superstructures we observe here. In contrast, the emergent bundles of $E c$ FtsZ at steady state extend longer than $2 \mu \mathrm{m}$, dimensions not reported in cells for $E$. coli FtsZ. While the patterns formed by E. coli FtsZ protofilaments provide insights into the effects of constraining gently curved dynamic filaments to a flat and fluid surface (RamirezDiaz et al., 2018), their relevance to understanding FtsZ assembly in vivo might thus be limited.

Although the intrinsic assembly properties of FtsZ from $C$. crescentus on SLBs differ from those of FtsZ from E. coli as reported here, the structures formed by each in cells are remarkably similar. It is possible that while $E c$ and $C c$ FtsZ behave differently under identical reaction conditions in vitro, the physiological context of FtsZ assembly within $E$. coli and $C$. crescentus cells might be vastly different. For example, factors such as membrane composition, $\mathrm{pH}$, and salt concentrations, particularly magnesium concentration, could affect surface concentration of polymers and/or their dynamics and thereby affect their superstructures. The differences in assembly we observe in vitro could reflect the divergent evolution of $E c$ and $C c$ FtsZ to suit their specific environments. Additionally, this also implicates species-specific regulatory factors in vivo in modifying the assembly properties of FtsZ to generate a Z-ring with the appropriate dynamics and structure to effect division. The repertoire of Z-ring associated proteins that affect protofilament bundling and/or turnover is vast and varies across species (Gueiros-Filho and Losick, 2002; Mohammadi et al., 2009; Goley et al., 2010; Galli and Gerdes, 2011; Durand-Heredia et al., 2012; Woldemeskel et al., 2017; Lariviere et al., 2018). For example, while FzlA, an essential protein that binds and assembles FtsZ filaments into helical bundles in vitro, is conserved in alpha-proteobacteria including $C$. crescentus, it is absent from other bacteria including $E$. coli (Goley et al., 2010; Lariviere et al., 2018). On the other hand, ZapC and ZapD, which induce bundling of $E$. coli FtsZ in vitro are not conserved in $C$. crescentus and other organisms. Moreover, while E. coli ZapA bundles E. coli FtsZ protofilaments (Low et al., 2004; Small et al., 2007; Mohammadi et al., 2009), C. crescentus ZapA has no appreciable effects on C. crescentus FtsZ protofilaments in vitro (Woldemeskel et al., 2017). Such differences in the availability and activity of FtsZ-bundling proteins could rectify the species-specific differences in the intrinsic higher order assembly of FtsZ we observe here to yield similar in vivo structures (Figure 1). Determining the effects of FtsZ-bundling proteins and other regulators of FtsZ assembly on the higher order assembly of protofilaments on SLBs will provide further insight into the regulation of Z-ring structure and dynamics in vivo.

An important variation in FtsZ across species is the length and sequence of the CTL (Vaughan et al., 2004). Whereas E. coli FtsZ has a CTL of 48 amino acids, $C$. crescentus 
FtsZ has a much longer CTL of 172 amino acids. Curiously, when high concentrations of $B$. subtilis FtsZ CTL variants were polymerized in solution and observed by cryo-electron microscopy, the minimum spacing between adjacent protofilaments was found to correlate with the presence and length of the CTL (Huecas et al., 2017). It is possible that the difference in the length and sequence of the CTL between E. coli FtsZ and C. crescentus FtsZ contributes to the differences in their emergent structures on SLBs in vitro by altering intrinsic lateral and longitudinal interactions. For example, $C$. crescentus CTL might be more effective in reducing lateral interaction in $C c$ FtsZ thereby preventing transient bundling required for forming the filamentous patterns observed for $E c$ FtsZ polymers. A thorough characterization of FtsZ polymerization on membranes across species would be crucial in understanding the contributions of the CTL and other intrinsic factors to speciesdependent differences in polymerization properties.

As demonstrated in this study and previous characterizations, the CTL plays an important role in preventing excess lateral interactions in $C$. crescentus (Sundararajan and Goley, 2017) and E. coli (Wang et al., 1997). Cc $\Delta$ CTL forms bundles in solution that can be observed on carbon-coated grids by electron microscopy (Sundararajan and Goley, 2017) or SLBs by TIRFM (Figure 3). We observe differences in the $\triangle$ CTL superstructures depending on whether the polymers were pre-formed in solution or directly assembled on the membrane. In the 1-inlet setup, the extended $\Delta \mathrm{CTL} / \Delta \mathrm{CTL}-\mathrm{MTS}$ copolymer bundles are cooperatively assembled in solution prior to being introduced into the flowcell and are maintained stably even after being recruited to the SLB surface. The slow turnover of $\Delta$ CTL/ $\Delta$ CTL-MTS polymers could limit the free monomer concentration and prevent the formation of new bundles on the membrane. On the other hand, in the 2-inlet setup, $\Delta$ CTL/ $\Delta$ CTL-MTS polymers likely assemble into bundles directly on the SLB surface following flow-stop near the laminar boundary. The immediate assembly of stable bundles of $\Delta$ CTL/ $\Delta$ CTL-MTS filaments closest to the protein/GTP channel interface generates a gradient of polymer concentration that is highest at the laminar boundary (Figure 4F). This gradient could ensure the continued assembly of polymers near the laminar boundary due to cooperative assembly and slow turnover of bundles. Since no such gradient is established in the 1-inlet setup, the bundles pre-formed in solution are dispersed during flow where they locally deplete monomers and eventually disappear due to photobleaching. Thus, being constrained to the membrane during assembly in the 2-inlet setup, $\Delta$ CTL/ $\Delta$ CTL-MTS polymers form uniform structures made of filament bundles that are retained at the laminar boundary due to slow turnover.

The CTL also appears to affect turnover of FtsZ polymers in C. crescentus and B. subtilis. Similar to $C c \Delta C T L$ (Sundararajan and Goley, 2017), Bs $\Delta$ CTL has reduced GTP hydrolysis rates and forms far fewer protofilaments (by electron microscopy) compared to $B s$ FtsZ (wt) at $2.5 \mathrm{mM} \mathrm{MgCl} 2$ and $50 \mathrm{mM} \mathrm{KCl}$ concentrations (Buske and Levin, 2013). Since neither $C c$ or $B s$ FtsZ form bundles under these conditions, it is possible that the contributions of the CTL to FtsZ turnover might be independent of its regulation of lateral interaction between protofilaments. Indeed, we postulated from our solution-based characterization of $C c$ FtsZ that the CTL influences the stability of longitudinal interactions between subunits, as well. Whether $B s \Delta \mathrm{CTL}$ would form bundles at higher $\mathrm{MgCl}_{2}$ and/or $\mathrm{KCl}$ concentrations similar to $C c \Delta$ CTL (Sundararajan and Goley, 2017) and Ec $\Delta$ CTL (Wang et al., 1997) is unclear. It 
is, however, interesting to note that $B s$ FtsZ has a short C-terminal Variable (CTV) region at its extreme $\mathrm{C}$-terminus that is a critical determinant of protofilament bundling (Buske and Levin, 2012). The CTV, which is also present in Ec FtsZ, but absent in $C c$ FtsZ, might contribute to species-dependent differences in FtsZ assembly properties.

In addition to elaborating on the structural differences previously observed by electron microscopy, we observe clear differences in dynamics between FtsZ and $\triangle \mathrm{CTL}$ using the approach described here. Our in vitro measurements of FtsZ dynamics on the membrane suggest that intrinsic dynamics of $C$. crescentus FtsZ are comparable to those of FtsZ from E. coli and $B$. subtilis. The time to attain half-maximum FRAP of $C$. crescentus FtsZ we observe ( 20s, Supplementary figure 2D) is similar to measurements of FRAP for E. coli FtsZ on supported lipid bilayers ( 10 s (Arumugam et al., 2014)), or in vivo ( 30 s (Stricker et al., 2002), $\sim 10 \mathrm{~s}$ (Anderson et al., 2004; Buss et al., 2015)). Similar recovery rates have been observed for B. subtilis FtsZ in vivo ( 10 s (Anderson et al., 2004)).). Moreover, our estimate of the rate of turnover of FtsZ polymers on the membrane is similar to previous estimations from bulk measurements in solution for $C c$ FtsZ ( 10 s, Milam and Erickson, 2013), Ec FtsZ (6 \pm 1 s, Chen and Erickson, 2005) and $B s$ FtsZ (10 \pm 2 s, Bisson-Filho et al., 2015).

In contrast to $C c$ FtsZ polymers, $C c \Delta \mathrm{CTL}$ polymers have a slower GTP hydrolysis rate (Sundararajan and Goley, 2017) and take proportionally longer to disassemble after GTP depletion (Figure 5D, Supplementary Figure 5D, 5E) or to recover after photobleaching (Figure 5E). These results confirm that the decrease in GTP hydrolysis rate observed for $C c$ $\triangle \mathrm{CTL}$ is directly linked to its turnover. The slow turnover of $\triangle \mathrm{CTL}$ suggests that the gradual disappearance of $\Delta C T L / \Delta C T L-M T S$ bundles (Figure 3B, Supplementary figure 3C) in the 1inlet setup is more likely due to faster photobleaching of the stable elongated structures rather than due to faster disassembly of bundles. Interestingly, mutants of FtsZ with similar (or reduced) GTP hydrolysis rates compared to $\triangle \mathrm{CTL}$ do not cause envelope bulging and cell lysis in vivo (Sundararajan et al., 2015) or affect polymer structure in vitro (Sundararajan and Goley, 2017). Therefore, we propose that the combined effects of the CTL on organization and turnover of protofilaments contribute to $\triangle \mathrm{CTL}$ 's lethal effects on cell wall metabolism in vivo.

Overall, our study provides the first in vitro characterization of polymer structure and dynamics on the membrane for FtsZ from a species other than E. coli. We have added spatio-temporal detail to the regulatory effects of the CTL on inter-filament interaction and turnover of $C$. crescentus FtsZ. While the current study uses an artificial membrane targeting sequence to constrain FtsZ polymerization to the membrane, expanding the study to include physiological membrane anchoring proteins such as FtsA and FzlC will be important future work. Furthermore, a large number of components of the division machinery dynamically interact with FtsZ, including those directly involved in peptidoglycan synthesis remodeling. The extension of the cell-free reconstitution system described here to investigate the interaction between FtsZ and the division machinery would greatly contribute to our understanding of the bacteria cell division process. 


\section{Experimental Procedures}

\section{Purification of proteins}

$E c$ His $_{6}$-FtsZ-venus-MTS was expressed for purification in E. coli Rosetta(DE3)pLysS cells using pET28C vector pEG658. All $C$. crescentus FtsZ variants (including $C c$ FtsZ-venusMTS - pEG717, WT FtsZ - pMT219, FtsZ-MTS - pEG1295, $\Delta$ CTL - pEG681, $\Delta$ CTLMTS - pEG1293, L14 - pEG723, L14-MTS - pEG1297, HnCTL - pEG676, HnCTL-MTS -pEG1296) used in this study were expressed for purification in E. coli Rosetta(DE3)pLysS cells using pET21a expression vectors (Supplementary Table 1). Nucleotide sequence information for previously unpublished plasmids are provided in Supplementary information. All FtsZ variants were purified using the previously published protocol for purifying $C$. crescentus FtsZ (Sundararajan et al., 2015; Sundararajan and Goley, 2017). Cells were induced for expression of FtsZ variants for 3 hours at $37^{\circ} \mathrm{C}$ at $\mathrm{OD} 600=1.0$ and pelleted following induction. The cell pellets were resuspended in lysis buffer $(50 \mathrm{mM}$ Tris$\mathrm{HCl}$ pH 8.0, $50 \mathrm{mM} \mathrm{KCl}, 1 \mathrm{mM}$ EDTA, 10\% glycerol, DNase I, $1 \mathrm{mM} \beta$-mercaptoethanol, 2 mM PMSF with cOmplete mini, EDTA-free protease inhibitor tablet (Roche)), and lysed using lysozyme treatment $\left(1 \mathrm{mg} \mathrm{mL}^{-1}\right)$ for 1 hour, followed by sonication to complete lysis. The lysate was then centrifuged at $6000 \times \mathrm{g}$ for 30 minutes to remove cell debris and the filtered supernatant was applied to an anion exchange column (HiTrap Q HP 5 mL, GE Life Sciences). Fractions containing the FtsZ variant were eluted using a linear gradient of $\mathrm{KCl}$ and were pooled. The FtsZ variant was then precipitated from the eluate using ammonium sulfate (20-35\% saturation depending on the FtsZ variant) and confirmed using electrophoresis (SDS-PAGE) and Coomassie staining. The ammonium sulfate precipitate was resuspended in FtsZ storage buffer (50 mM HEPES-KOH pH 7.2, 0.1 mM EDTA, 50 $\mathrm{mM} \mathrm{KCl}, 0.1 \mathrm{mM}$ EDTA, $1 \mathrm{mM} \beta$-mercaptoethanol, $10 \%$ glycerol) and purified further using size-exclusion chromatography (Superdex 200 10/300 GL, GE Life Sciences). The purified protein in FtsZ storage buffer was then snap frozen in liquid nitrogen and stored at $-80{ }^{\circ} \mathrm{C}$.

$E c$ FtsZ-venus-MTS was expressed and purified as His $6^{-S U M O-E c F t s Z-v e n u s-M T S ~ i n ~} E$. coli Rosetta(DE3)pLysS cells using pTB146 vector from pEG659. Protein expression was induced using $0.5 \mathrm{mM}$ IPTG for 4 hours at $37{ }^{\circ} \mathrm{C}$ at OD600 $=1.0$. Cells were pelleted and resuspended in His-column buffer $(50 \mathrm{mM}$ Tris- $\mathrm{HCl} \mathrm{pH} \mathrm{7.2,300} \mathrm{mM} \mathrm{KCl,} 20 \mathrm{mM}$ Imidazole, 10\% glycerol) containing DNase I, $1 \mathrm{mM} \beta$-mercaptoethanol, $2 \mathrm{mM}$ PMSF, 2.5 $\mathrm{mM} \mathrm{MgCl} 2$. Cells were then lysed by lysozyme treatment and sonication as mentioned above. His $_{6}$-SUMO-FtsZ-venus-MTS was then purified from the clarified lysate using HisTrap FF $1 \mathrm{ml}$ column (GE Life Sciences) by eluting with $300 \mathrm{mM}$ imidazole. The His $6^{-}$ SUMO- tag was cleaved overnight using SUMO protease, His $_{6}$-Ulp1, at a 100-fold molar excess and simultaneously dialyzed into His-column buffer. Untagged $E c$ FtsZ-venus-MTS was separated from the uncleaved His $_{6}$-SUMO-FtsZ-venus-MTS, the cleaved His 6 -SUMOtag, and His $_{6}$-Ulp1 by passage over HisTrap $1 \mathrm{ml}$ column once again and collecting the flow through. Untagged $E c$ FtsZ-venus-MTS was further purified and buffer-exchanged into FtsZ storage buffer using size exclusion chromatography (Superdex 200 10/300 GL, GE Life Sciences). The purified $E c$ FtsZ-venus-MTS protein was then snap frozen in liquid nitrogen and stored at $-80{ }^{\circ} \mathrm{C}$. 
After purification, FtsZ, $\Delta$ CTL, L14 and HnCTL were subjected to Alexa488 dye labeling using Alexa Fluor 488 C5 Maleimide (ThermoFisher Scientific) reagent and the manufacturer's protocol. Purified FtsZ or FtsZ variant was treated for 1 hour with a 10 times molar excess of DTT in FtsZ storage buffer to reduce the only cysteine residue in FtsZ, followed by incubation with at least 10 molar excess of Alexa Fluor 488 dye solution for 2 hours at room temperature or overnight at $4 \mathrm{C}$. Following incubation, a 20 times molar excess of $\beta$-mercaptoethanol was added to quench excess reagent in the reaction and the labeled protein was purified using size-exclusion chromatography (Superdex 200 10/300 GL, GE Life Sciences). The fluorescent fractions were pooled, concentrated and the stored at $-80{ }^{\circ} \mathrm{C}$. Prior to freezing, the labeling efficiency (as percentage labeled) was determined using absorption measurements. $\triangle \mathrm{CTL}$ had the lowest labeling efficiency (6\%) compared to other FtsZ variants. Hence, all experiments involving comparisons of $\Delta \mathrm{CTL}$ to other FtsZ variants were performed with 6\% labeled FtsZ variant in the final reaction.

\section{Preparation of flow cells}

One- and two-inlet flow cells were prepared as described previously with a few modifications (Vecchiarelli et al., 2016). Quartz glass slides with drilled one or two inlet holes and one outlet hole each (Esco products) were cleaned by washing overnight in NOCHROMIX glass cleaner (Sigma), rinsed with ultrapure water, air dried, and treated with low-power plasma cleaning in the presence of argon and oxygen. A rectangular piece of 25$\mu \mathrm{m}$ thick acrylic transfer tape $(3 \mathrm{M})$ of $\sim 5 \mathrm{~cm} \times \sim 3.5 \mathrm{~cm}$ was cut to demarcate the required chamber dimensions (for one-inlet flow cell, rectangular region of $4 \mathrm{~mm}$ wide $\times 3 \mathrm{~cm}$ long was cut out, for two-inlet flow cell, y-shaped region with a uniform width of $4 \mathrm{~mm}$ was cut out). The tape was placed between the glass slide and cover slip. Nanoports (Upchurch) adapters were attached to the slides above the holes with optical adhesive. The flow cell was then baked at $65^{\circ} \mathrm{C}$ for 1 hour.

We often observed that FtsZ protofilaments were preferentially recruited or excluded along parallel straight lines on the SLBs. We hypothesized that this was due to scratches along the glass surface, giving rise to extended regions of curved membrane. While we observed these ordered linear patterns for $E_{c}$ His $_{6}$-FtsZ-venus-MTS, $C c$ FtsZ-venus-MTS as well as with Alexa488-labeled FtsZ, they were most obvious in experiments using partially labeled FtsZ (for example, FtsZ (35\% FtsZ-Alexa488)/FtsZ-MTS). To avoid loss in signal-to-noise in imaging regions adjacent to scratches and to prevent possible artifacts, we treated the glass slides with hydrofluoric acid (HF) to remove scratches on the surface in all our experiments that involved Alexa-labeled FtsZ variant. Glass slides were incubated in 20\% HF solution for 2 minutes, and then washed by immersing in $100 \mathrm{mM} \mathrm{CaCl}_{2}$ solution bath, and rinsed well with water prior to wash with NOCHROMIX. HF treatment of glass slides eliminated the appearance of parallel straight lines.

\section{Preparation of SUVs}

Minimum synthetic lipid mixtures were made using 33:67 or 20:80 combinations of 1,2dioleoyl-sn-glycero-3-[phospho-rac-(1-glycerol)] (DOPG; Cat. No. 840475, Avanti) and 1,2dioleoyl-sn-glycero-3-phosphocholine (DOPC; Cat. No. 850375, Avanti). The purchased synthetic lipids resuspended in chloroform at $25 \mathrm{mg} \mathrm{mL}^{-1}$ were mixed to appropriate ratios 
in glass tubes pre-rinsed with chloroform. After thorough mixing, the lipid mixture was dried by evaporating chloroform using dry $\mathrm{N}_{2}$ gas with constant rotation to make a thin layer of dry lipids and was dried further in a SpeedVac Concentrator (Savant) for 1 hour at $42{ }^{\circ} \mathrm{C}$ initially and 1 hour at room temperature subsequently. The dried lipid mixture was resuspended by vortexing in degassed TK150 buffer ( $25 \mathrm{mM}$ Tris-HCl, $\mathrm{pH} 7.4,150 \mathrm{mM}$ $\mathrm{KCl}$ ) to a lipid concentration of $5 \mathrm{mg} \mathrm{mL}^{-1}$ and was incubated overnight in the dark at room temperature in an $\mathrm{N}_{2}$ atmosphere ( $\mathrm{N} 2$ box). The next day, the aqueous resuspension of lipids was mixed thoroughly by vortexing and was transferred to polystyrene tubes. The resuspension was sonicated at $23{ }^{\circ} \mathrm{C}$ immersed in a water bath sonicator (Qsonica model \#Q700A) at $70 \mathrm{~W}$ for 5 minutes (30 s per pulse with $10 \mathrm{~s}$ rest) until the turbid resuspension (made of multilamellar vesicles of non-uniform dimensions) turned translucent and blueshifted (corresponding to $\sim 100 \mathrm{~nm}$ small unilamellar vesicle or SUVs). Under $\mathrm{N}_{2}$ atmosphere, the sonicate was then filtered using 0.2 micron filter to purify SUVs, aliquoted and stored in Teflon-coated and parafilm-sealed glass vials at $4{ }^{\circ} \mathrm{C}$. SUV stocks were used within 5 weeks from the date of preparation.

\section{Preparation of SLBs}

Supported lipid bilayers were made by triggering attachment of SUVs to plasma cleaned glass slide surface within the flow cell by incubation with $5 \mathrm{mM} \mathrm{MgCl} 2$ in TK150 buffer for 1 hour at $37^{\circ} \mathrm{C}$. The flow cell was first equilibrated by flowing in TK150 buffer $\mathrm{pH} 7.4$ containing $5 \mathrm{mM} \mathrm{MgCl}_{2}$ (TK150M5). The SUVs from the stock solution were diluted to 0.5 $\mathrm{mg} \mathrm{mL}^{-1}$ in TK150M5 buffer and the solution was incubated at $37^{\circ} \mathrm{C}$ for 5 minutes. $300 \mu \mathrm{L}$ of the SUV solution in TK150M5 was then flowed in at $10 \mu \mathrm{L} \mathrm{min}{ }^{-1}$ into the flow cell maintained at $37^{\circ} \mathrm{C}$. The flow cell was then incubated for 1 hour at $37^{\circ} \mathrm{C}$ to allow fusion of SUVs to form supported lipid bilayers. The excess SUVs were removed by flowing in 500 $\mu \mathrm{L}$ of TK150M5 buffer. The flow cells with SLBs were then equilibrated for subsequent experiments by flowing in appropriate reaction buffers. The flow cells were maintained at $37^{\circ} \mathrm{C}$ until mounting on the microscope stage and were maintained above $24{ }^{\circ} \mathrm{C}$ during experiments to maintain membrane fluidity by avoiding phase transition of SLBs at lower temperatures.

\section{FtsZ polymerization reactions}

Imaging experiments involving $E c$ His $_{6}$-FtsZ-venus-MTS, $E c$ FtsZ-venus-MTS or $C c$ FtsZvenus-MTS were performed in HMKKG FtsZ polymerization buffer (50 mM HEPES-KOH pH 7.2, $5 \mathrm{mM} \mathrm{MgCl}_{2}, 150 \mathrm{mM} \mathrm{KCl}, 50 \mathrm{mM} \mathrm{K}\left(\mathrm{CH}_{3} \mathrm{CO}_{2}\right) 10 \%$ glycerol) containing $1 \%$ casein (w/v) and $0.5 \mathrm{mg} \mathrm{mL}^{-1}$ ascorbate using $2 \mu \mathrm{M}$ FtsZ-venus-MTS incubated with $2 \mathrm{mM}$ GTP for 30 minutes prior to flowing into flow cells with SLBs made from 33\% DOPG, 67\% DOPC SUVs.

Imaging experiments involving FtsZ/FtsZ-MTS, $\Delta$ CTL/ $\Delta$ CTL-MTS, L14/L14-MTS or HnCTL/HnCTL-MTS were performed in HEK300 FtsZ polymerization buffer containing 50 $\mathrm{mM}$ HEPES-KOH pH 8.0, $0.1 \mathrm{mM}$ EDTA, $10 \mathrm{mM} \mathrm{MgCl}_{2}$ (unless otherwise mentioned), $300 \mathrm{mM} \mathrm{KCl}$ with $1 \%$ casein (w/v) and $0.5 \mathrm{mg} \mathrm{mL}^{-1}$ ascorbate incubated with $2 \mathrm{mM}$ GTP for 5 minutes as required prior to flowing into the flow cells with SLBs made from $20 \%$ DOPG, $80 \%$ DOPC SUVs. These reaction conditions and membrane composition were 
determined to be optimum for reducing non-specific interaction of FtsZ polymers with the membrane in the absence of the MTS to improve signal-to-noise ratio. The protein mixtures were filtered using centrifugal filters prior to addition of nucleotide to remove non-specific protein aggregates on SLBs.

\section{TIRF microscopy, imaging and analysis}

Illumination and imaging were performed using instrumentation described previously (Vecchiarelli et al., 2016). All TIRFM experiments were performed on flow cell mounted on an Eclipse TE200E microscope (Nikon) with a prism placed on top of the glass slide (with oil, $\mathrm{n}=1.49$, between prism and glass slide) and imaged through the coverslip (bottom) through Plan Apo 10X (NA = 0.45, air) or Plan Apo 100X (NA = 1.4, oil immersed) objectives (Nikon). An Andor DU-879E camera was used for image acquisition with the following settings: digitizer $-3 \mathrm{MHz}$ (14 bit-gray scale), preamplifier gain -5.2 , vertical shift speed, $2 \mathrm{MHz}$, vertical clock range - normal, electron-multiplying gain - 40, EM CCD temperature $--98{ }^{\circ} \mathrm{C}$, baseline clamp - ON, exposure time $-100 \mathrm{~ms}$.

The excitation at $488 \mathrm{~nm}$ for FtsZ-venus-MTS and Alexa fluor 488 labeled FtsZ was provided using a $488 \mathrm{~nm}$ diode-pumped solid-state laser (Sapphire, Coherent) at $8 \mu \mathrm{W}$. TIRF illumination had a Gaussian shape in the field of view that could be broadened using a diffuser at the incident beam. Images were acquired in regions of uniform illumination profile to improve signal to noise.

Images were acquired at $0.5,2$ or 5 seconds per frame as mentioned in movie legends using Metamorph 7 (Molecular Devices) to make time-lapse movies in ImageJ (National Institute of Health). Movies were made from $150 \mathrm{px} \times 150 \mathrm{px}$ or $200 \mathrm{px} \times 200 \mathrm{px}$ regions of interest (ROIs) that were cropped from $512 \mathrm{px} \times 512 \mathrm{px}$ fields of view and brightness/contrast adjusted, by enhancing contrast by saturating the highest $2 \%$ of intensities for each frame. The same brightness adjustment was applied to each frame. Movies were then converted to Audio Video Interleave format (.avi). Unless specified, time lapse image stacks were sped up to 20 times to make the movies (each second of the movie equals 20 seconds in real time). The time at the upper-left corner of movies represent time in 'minute:seconds' format. Representative still images for figures were made from $5 \mathrm{~s}$ time averages at specified time points (i.e. 10 frame time average for 0.5 seconds per frame acquisition, 5 frame time average for 2 seconds per frame acquisition, and 2 frame time average for 5 seconds per frame acquisition), to improve signal to noise.

Dimensions of fluorescent clusters of $E$. coli His $_{6}$-FtsZ-venus-MTS and $C$. crescentus FtsZvenus-MTS and of bundles of E. coli His $_{6}$-FtsZ-venus-MTS on SLBs in Figures $1 \mathrm{D}$ and $1 \mathrm{E}$ were estimated using line-scans across the short axis (width) or long axis (length) of these structures. The short and long axes were obvious mainly for E. coli His $_{6}$-FtsZ-venus-MTS after 5 minutes on the SLB (Figure 1D). For circular or amorphous clusters, the shortest distance across the cluster was estimated. Fluorescent profiles were measured along lines drawn through the structures. The distances between points of half-maximum intensity (full width at half-maximum) were determined from polynomial fits to the fluorescence profiles that were generated using Graphpad Prism Software (Graphpad Software Inc., La Jolla, CA). 
Intensity plot profiles were measured as averages of fluorescence intensities in regions of interest (entire frame $-150 \mathrm{px} \times 150 \mathrm{px}, 200 \mathrm{px} \times 200 \mathrm{px}$, FRAP $-40 \mathrm{px} \times 40 \mathrm{px}$ ROI within photobleached region, $\Delta$ CTL bundles - minimum rectangular ROIs, approximately 8 $\mathrm{px} \times 15 \mathrm{px}$, around bright filamentous structures) per frame.

Movies showing flow-stop specific intensity changes in two-inlet flow setup in figure 4 were acquired using a 10X objective. Corresponding kymographs were obtained at line (spline width $4 \mathrm{px}$ ) perpendicular to the direction of flow. $30 \mathrm{px} \times 30 \mathrm{px}$ ROIs were used for measuring corresponding fluorescence intensity profiles over time in these experiments.

\section{GTP depletion experiments}

Rapid GTP depletion to induce disassembly of FtsZ or $\triangle$ CTL polymers on SLBs were performed using the two-inlet setup as described in Figure 4. The rate of disassembly and half-lives of the polymers on SLBs after GTP depletion (Figure 5D) were estimated using exponential decay fits to fluorescence intensity profiles over time by TIRFM at 100x magnification averaged over ROIs of $40 \mathrm{px} \times 40 \mathrm{px}$ ROI.

\section{Photobleaching experiments}

Fluorescence recovery after photobleaching (FRAP) experiments were performed using high power laser applied for 3 seconds on the SLBs through the objective lens using $\sim 6$ times the intensity used for the incident light for TIRF, while momentarily pausing image acquisition. We observe a minimum $40 \%$ loss in fluorescence immediately following photobleaching in our FRAP experiments. Time to half-maximum FRAP were estimated using one-phase association curves fit to fluorescence intensity profiles of individual replicates.

\section{Supplementary Material}

Refer to Web version on PubMed Central for supplementary material.

\section{Acknowledgements}

We would like to thank the members of the Goley lab - Elizabeth Meier, PJ Lariviere, Selam Woldemeskel, Anant Bhargava, Allison Daitch, and Chris Mahone - and the Mizuuchi lab - James Taylor and Michiyo Mizuuchi - for helpful discussions that informed design, optimization and analyses described in this work. We would also like to thank Jie Xiao, Xinxing Yang, and Keir Neuman for their suggestions for quantifying FtsZ dynamics on SLBs. Funding for this work was provided by the NIH through R01GM108640 (to E.D.G.) and the intramural research fund for National Institute of Diabetes and Digestive and Kidney Diseases (to K.M).

\section{References}

Aaron M, Charbon G, Lam H, Schwarz H, Vollmer W, and Jacobs-Wagner C (2007) The tubulin homologue FtsZ contributes to cell elongation by guiding cell wall precursor synthesis in Caulobacter crescentus. Molecular Microbiology 64: 938-952. [PubMed: 17501919]

Anderson DE, Gueiros-Filho FJ, and Erickson HP (2004) Assembly dynamics of FtsZ rings in Bacillus subtilis and Escherichia coli and effects of FtsZ-regulating proteins. Journal of Bacteriology 186: 5775-5781. [PubMed: 15317782]

Arumugam S, Chwastek G, Fischer-Friedrich E, Ehrig C, Mönch I, and Schwille P (2012) Surface topology engineering of membranes for the mechanical investigation of the tubulin homologue FtsZ. Angew Chem Int Ed Engl 51: 11858-11862. [PubMed: 22936525] 
Arumugam S, Petrašek Z, and Schwille P (2014) MinCDE exploits the dynamic nature of FtsZ filaments for its spatial regulation. Proceedings of the National Academy of Sciences 111: E1192200.

Bisson-Filho AW, Discola KF, Castellen P, Blasios V, Martins A, Sforca ML, et al. (2015) FtsZ filament capping by MciZ, a developmental regulator of bacterial division. Proceedings of the National Academy of Sciences 112: E2130-E2138.

Bisson-Filho AW, Hsu Y-P, Squyres GR, Kuru E, Wu F, Jukes C, et al. (2017) Treadmilling by FtsZ filaments drives peptidoglycan synthesis and bacterial cell division. Science 355: 739-743. [PubMed: 28209898]

Buske PJ, and Levin PA (2012) Extreme C terminus of bacterial cytoskeletal protein FtsZ plays fundamental role in assembly independent of modulatory proteins J Biol Chem 287: 10945-10957. [PubMed: 22298780]

Buske PJ, and Levin PA (2013) A flexible C-terminal linker is required for proper FtsZ assembly in vitro and cytokinetic ring formation in vivo. Molecular Microbiology 89: 249-263. [PubMed: 23692518]

Buss J, Coltharp C, Shtengel G, Yang X, Hess H, and Xiao J (2015) A Multi-layered Protein Network Stabilizes the Escherichia coli FtsZ-ring and Modulates Constriction Dynamics. PLoS Genet 11: e1005128. [PubMed: 25848771]

Chen Y, and Erickson HP (2005) Rapid in vitro assembly dynamics and subunit turnover of FtsZ demonstrated by fluorescnce resonance energy transfer. J Biol Chem 280: 22549-22554. [PubMed: 15826938]

Durand-Heredia J, Rivkin E, Fan G, Morales J, and Janakiraman A (2012) Identification of ZapD as a Cell Division Factor That Promotes the Assembly of FtsZ in Escherichia coli. Journal of Bacteriology 194: 3189-3198. [PubMed: 22505682]

Erickson HP, Anderson DE, and Osawa M (2010) FtsZ in bacterial cytokinesis: cytoskeleton and force generator all in one. Microbiol Mol Biol Rev 74: 504-528. [PubMed: 21119015]

Fu G, Huang T, Buss J, Coltharp C, Hensel Z, and Xiao J (2010) In Vivo Structure of the E. coli FtsZring Revealed by Photoactivated Localization Microscopy (PALM). PLoS ONE 5: e12680. [PubMed: 20856929]

Galli E, and Gerdes K (2011) FtsZ-ZapA-ZapB Interactome of Escherichia coli. Journal of Bacteriology 194: 292-302. [PubMed: 22056926]

Gardner KAJA, Moore DA, and Erickson HP (2013) The C-terminal linker of Escherichia coli FtsZ functions as an intrinsically disordered peptide. Molecular Microbiology 89: 264-275. [PubMed: 23714328]

Goley ED, Dye NA, Werner JN, Gitai Z, and Shapiro L (2010) Imaging-Based Identificationof a Critical Regulator of FtsZ Protofilament Curvature in Caulobacter. Molecular Cell 39: 975-987. [PubMed: 20864042]

Gueiros-Filho FJ, and Losick R (2002) A widely conserved bacterial cell division protein that promotes assembly of the tubulin-like protein FtsZ. Genes \& Development 16: 2544-2556. [PubMed: 12368265]

Holden SJ, Pengo T, Meibom KL, Fernandez Fernandez C, Collier J, and Manley S (2014) High throughput 3D super-resolution microscopy reveals Caulobacter crescentus in vivo Z-ring organization. Proceedings of the National Academy of Sciences 111: 4566-4571.

Huecas S, Ramírez-Aportela E, Vergoñós A, Núñez-Ramírez R, Llorca O, Díaz JF, et al. (2017) SelfOrganization of FtsZ Polymers in Solution Reveals Spacer Role of the Disordered C-Terminal Tail. Biophysj 113: 1831-1844.

Lariviere PJ, Szwedziak P, Mahone CR, Löwe J, and Goley ED (2018) FzlA, an essential regulator of FtsZ filament curvature, controls constriction rate during Caulobacter division. Molecular Microbiology 107: 180-197. [PubMed: 29119622]

Li Z, Trimble MJ, Brun YV, and Jensen GJ (2007) The structure of FtsZ filaments in vivo suggests a force-generating role in cell division. The EMBO Journal 26: 4694-4708. [PubMed: 17948052]

Loose M, and Mitchison TJ (2014) The bacterial cell division proteins FtsA and FtsZ self-organize into dynamic cytoskeletal patterns. Nat Cell Biol 16: 38-46. [PubMed: 24316672] 
Low HH, Moncrieffe MC, and Löwe J (2004) The crystal structure of ZapA and its modulation of FtsZ polymerisation. J Mol Biol 341: 839-852. [PubMed: 15288790]

Meier EL, and Goley ED (2014) Form and function of the bacterial cytokinetic ring. Curr Opin Cell Biol 26: 19-27. [PubMed: 24529242]

Milam SL, and Erickson HP (2013) Rapid in vitro assembly of Caulobacter crescentus FtsZ protein at pH 6.5 and 7.2. J Biol Chem 288: 23675-23679. [PubMed: 23824192]

Mohammadi T, Ploeger GEJ, Verheul J, Comvalius AD, Martos A, Alfonso C, et al. (2009) The GTPase Activity of Escherichia coliFtsZ Determines the Magnitude of the FtsZ Polymer Bundling by ZapA in Vitro. Biochemistry 48: 11056-11066. [PubMed: 19842714]

Mukherjee A, and Lutkenhaus J (1999) Analysis of FtsZ assembly by light scattering and determination of the role of divalent metal cations. Journal of Bacteriology 181: 823-832. [PubMed: 9922245]

Oliva MA, Huecas S, Palacios JM, Martín-Benito J, Valpuesta JM, and Andreu JM (2003) Assembly of archaeal cell division protein FtsZ and a GTPase-inactive mutant into doubl-strand filaments. J Biol Chem 278: 33562-33570. [PubMed: 12807911]

Osawa M, and Erickson HP (2011) Inside-out Z rings--constriction with and without GTP hydrolysis. Molecular Microbiology 81: 571-579. [PubMed: 21631604]

Osawa M, and Erickson HP (2013) Liposome division by a simple bacterial division machinery. Proceedings of the National Academy of Sciences 110: 11000-11004.

Osawa M, Anderson DE, and Erickson HP (2008) Reconstitution of contractile FtsZ rings in liposomes. Science 320: 792-794. [PubMed: 18420899]

Osawa M, Anderson DE, and Erickson HP (2009) Curved FtsZ protofilaments generate bending forces on liposome membranes. The EMBO Journal 28: 3476-3484. [PubMed: 19779463]

Popp D, Iwasa M, Narita A, Erickson HP, and Maéda Y (2009) FtsZ condensates: an in vitro electron microscopy study. Biopolymers 91: 340-350. [PubMed: 19137575]

Ramirez-Diaz D, Garcia-Soriano DA, Raso A, Mücksch J, Feingold M, Rivas G, and Schwille P (2018) Treadmilling analysis reveals new insights into dynamic FtsZ ring architecture. PLoS Biol 16: e2004845. [PubMed: 29775478]

Redick SD, Stricker J, Briscoe G, and Erickson HP (2005) Mutants of FtsZ targeting the protofilament interface: effects of cell division and GTPase activity. Journal of Bacteriology 187: 2727-2736. [PubMed: 15805519]

Small E, Marrington R, Rodger A, Scott DJ, Sloan K, Roper D, et al. (2007) FtsZ polymer-bundling by the Escherichia coli ZapA orthologue, YgfE, involves a conformational change in bound GTP. J Mol Biol 369: 210-221. [PubMed: 17428494]

Stricker J, Maddox P, Salmon ED, and Erickson HP (2002) Rapid assembly dynamics of the Escherichia coli FtsZ-ring demonstrated by fluorescence recovery after photobleaching. Proc Natl Acad Sci USA 99: 3171-3175. [PubMed: 11854462]

Sundararajan K, and Goley ED (2017) The intrinsically disordered C-terminal linker of FtsZ regulates protofilament dynamics and superstructure in vitro. Journal of Biological Chemistry 292: 2050920527. [PubMed: 29089389]

Sundararajan K, Miguel A, Desmarais SM, Meier EL, Huang KC, and Goley ED (2015) The bacterial tubulin FtsZ requires its intrinsicallydisordered linker to direct robust cell wallconstruction. Nature Communications 6: 7281.

Szwedziak P, Wang Q, Bharat TAM, Tsim M, and Löwe J (2014) Architecture of the ring formed by the tubulin homologue FtsZ in bacterial cell division. Elife 3: e04601. [PubMed: 25490152]

Vaughan S, Wickstead B, Gull K, and Addinall SG (2004) Molecular evolution of FtsZ protein sequences encoded within the genomes of archaea, bacteria, and eukaryota. J Mol Evol 58: 19-29. [PubMed: 14743312]

Vecchiarelli AG, Li M, Mizuuchi M, Hwang LC, Seol Y, Neuman KC, and Mizuuchi K (2016) Membrane-bound MinDE complex acts as a toggle switch that drives Min oscillation coupled to cytoplasmic depletion of MinD. Proceedings of the National Academy of Sciences 113: E14791488.

Wang X, Huang J, Mukherjee A, Cao C, and Lutkenhaus J (1997) Analysis of the interaction of FtsZ with itself, GTP, and FtsA. Journal of Bacteriology 179: 5551-5559. [PubMed: 9287012] 
Woldemeskel SA, McQuillen R, Hessel AM, Xiao J, and Goley ED (2017) A conserved coiled-coil protein pair focuses the cytokinetic Z-ring in Caulobacter crescentus. Molecular Microbiology 76: 173.

Yang X, Lyu Z, Miguel A, McQuillen R, Huang KC, and Xiao J (2017) GTPase activity-coupled treadmilling of the bacterial tubulin FtsZ organizes septal cell wall synthesis. Science 355: 744747. [PubMed: 28209899] 
A
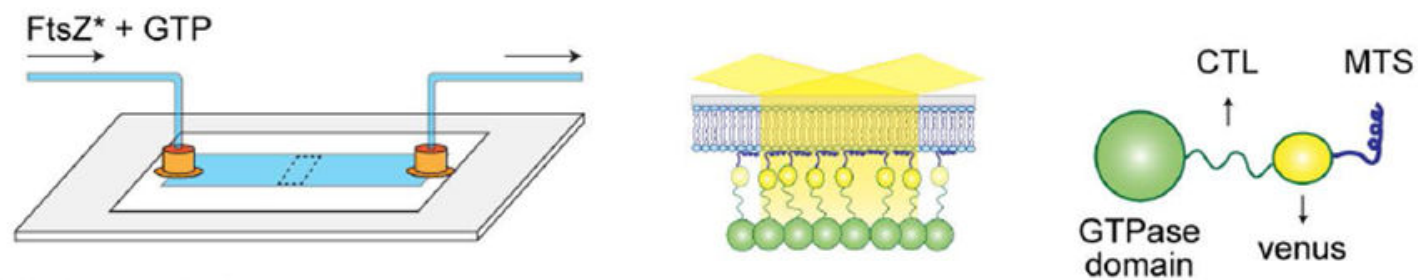

B Ec FtsZ-venus-MTS
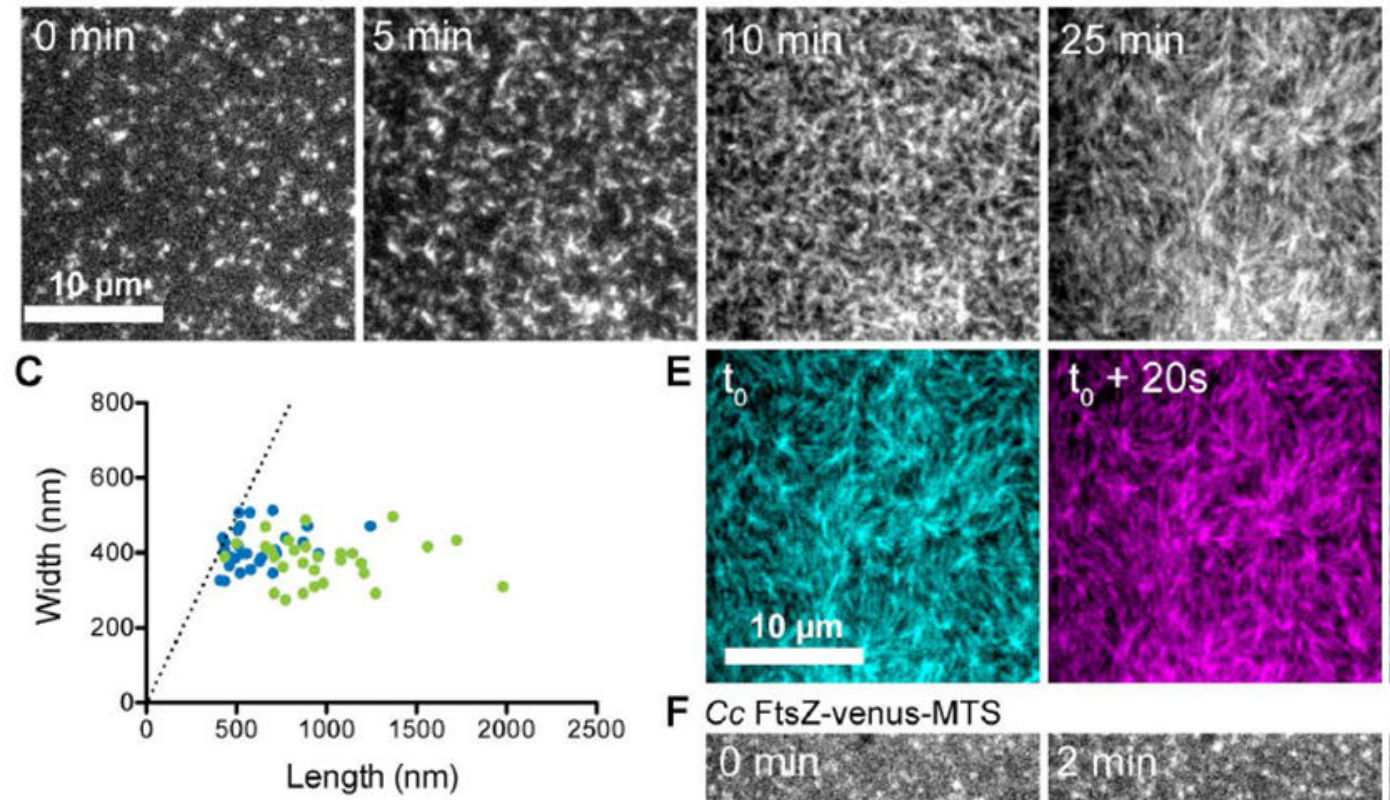

F Cc FtsZ-venus-MTS
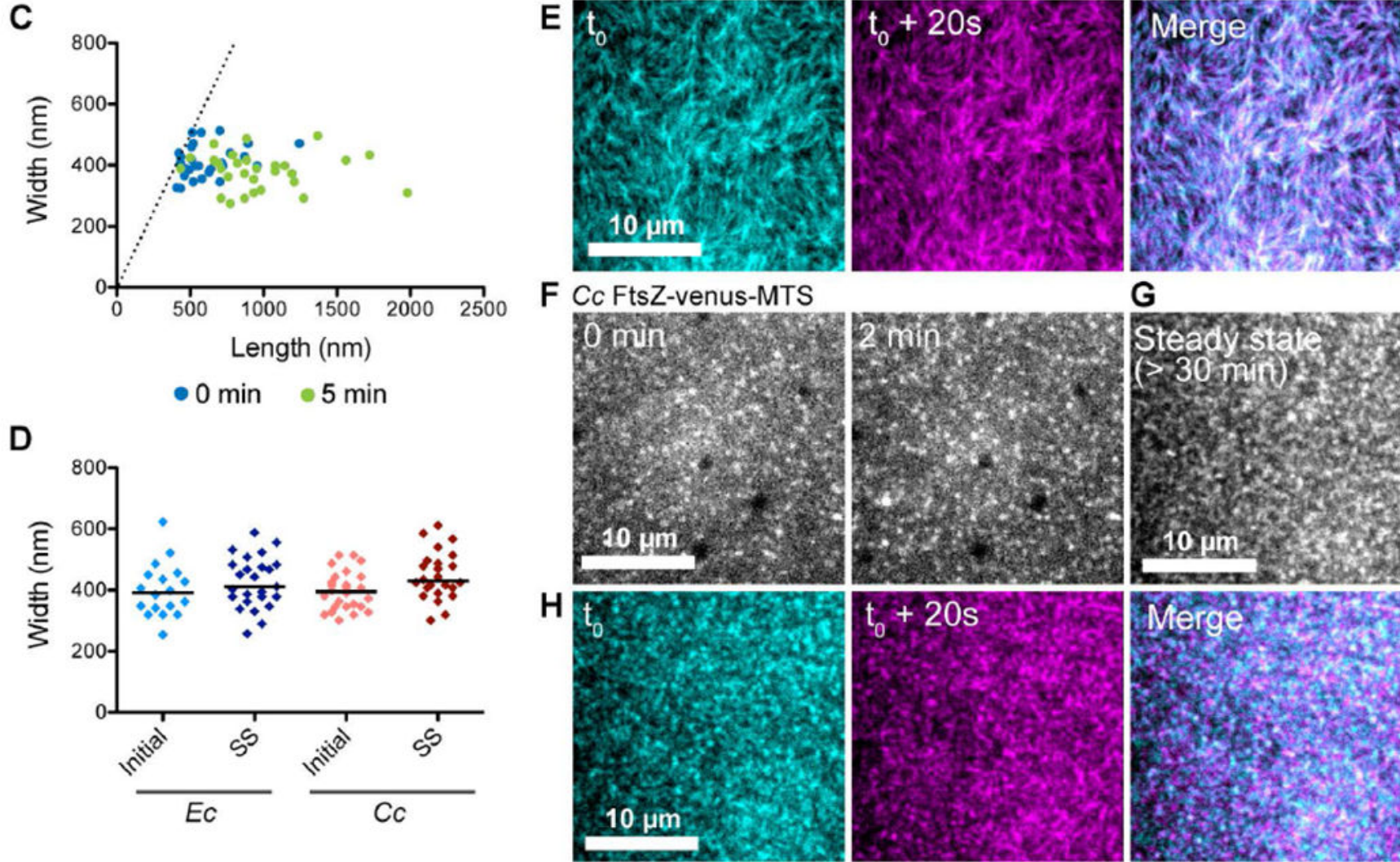

G

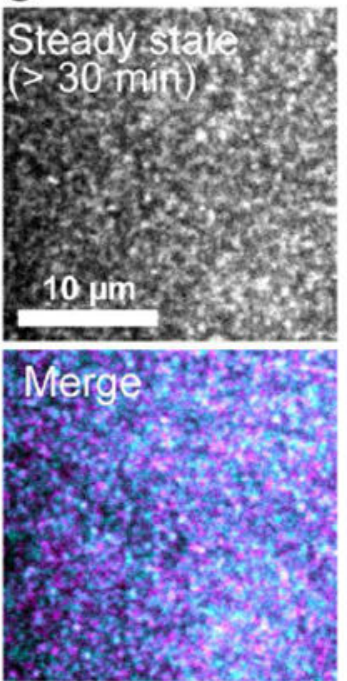

Figure 1.

FtsZ protofilaments assemble as dynamic clusters on SLBs that form species-specific superstructures. A. Schematic describing the flow cell setup used for imaging FtsZ polymer assembly. FtsZ* (FtsZ-venus-MTS) incubated with GTP is flowed into the flow cell. FtsZvenus-MTS protofilaments are recruited to the membrane through the MTS and are brought into the evanescent field of TIRF. B. Contrast enhanced TIRFM images showing structures formed by $2 \mu \mathrm{M} \mathrm{Ec}$ His $_{6}$-FtsZ-venus-MTS preincubated with $2 \mathrm{mM}$ GTP for 30 minutes and introduced into flow cell (at $5 \mu \mathrm{L}$ minute ${ }^{-1}$ ) with the SLB composed of $33 \%$ DOPG and 
67\% DOPC lipids. Time on the images indicates approximate time passed after the initiation of flow. C. Plot showing width (distance along short axis) and length (distance along long axis) of clusters formed at 0 minutes (blue) and 5 minutes (green) for experiment shown in B. Dotted line indicates the identity line (width $=$ length). D. Widths of clusters or bundles formed by $E$. coli His $_{6}$-FtsZ-venus-MTS or $C$. crescentus FtsZ-venus-MTS at initial time point (time $=0$ minutes) and at steady state (time $\geq 30$ minutes). Line indicates median. $\mathbf{E}$. Individual frames and merged images showing overlay of structures formed by $E_{c} \mathrm{His}_{6^{-}}$ FtsZ-venus-MTS at steady state spaced 20 seconds apart (cyan - time ' $t_{0}$ ', magenta - time ' $\mathrm{t}_{0}+20$ seconds', white regions in the merged image represent colocalization of signal) F.\& G. Contrast enhanced TIRFM images showing structures formed by $1.8 \mu \mathrm{M} \mathrm{Cc}$ FtsZ-venusMTS preincubated with $2 \mathrm{mM}$ GTP for 30 minutes and flowed into flow cell (at $5 \mu \mathrm{L}$ minute ${ }^{-1}$ ) with the SLB composed of 33\% DOPG and 67\% DOPC lipids. Time on the images indicates approximate time passed after the initiation of flow. G. Steady state structures formed by $C c$ FtsZ-venus-MTS after flow was stopped. H. Individual frames and merged images showing overlay of structures formed by $C c$ FtsZ-venus-MTS at steady state spaced 20 seconds apart (cyan - time ' $t_{0}$ ', magenta - time ' $t_{0}+20$ seconds', white regions in the merged image represent colocalization of signal). Scale bar - $10 \mu \mathrm{m}$. Reaction buffer contains $50 \mathrm{mM}$ HEPES pH 7.3, $5 \mathrm{mM} \mathrm{Mg}\left(\mathrm{CH}_{3} \mathrm{COO}\right)_{2}, 300 \mathrm{mM} \mathrm{KCH}{ }_{3} \mathrm{COO}, 50 \mathrm{mM} \mathrm{KCl}$, $10 \%$ glucose, $0.1 \mathrm{mg} \mathrm{mL}^{-1}$ casein (blocking agent). 
A

(i)
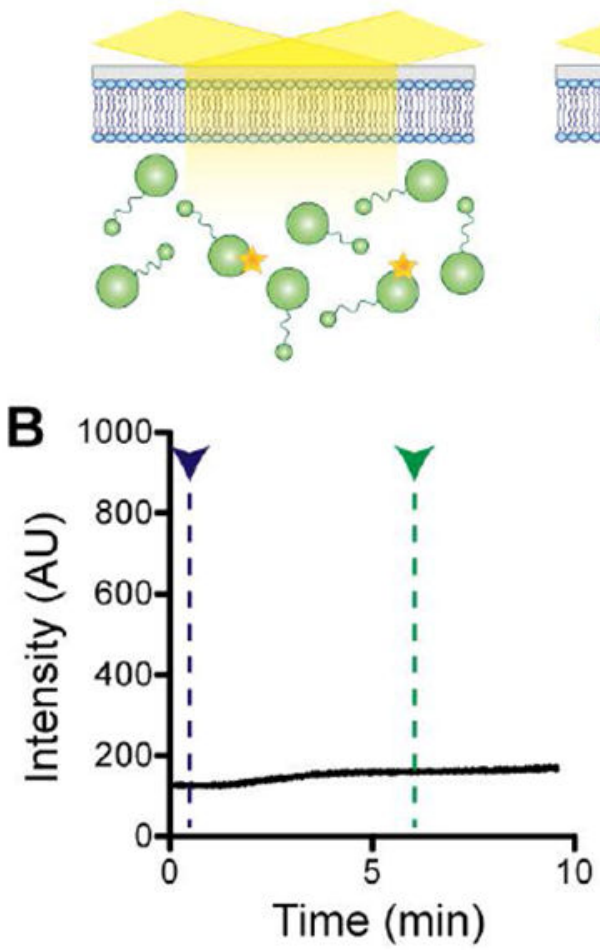

D

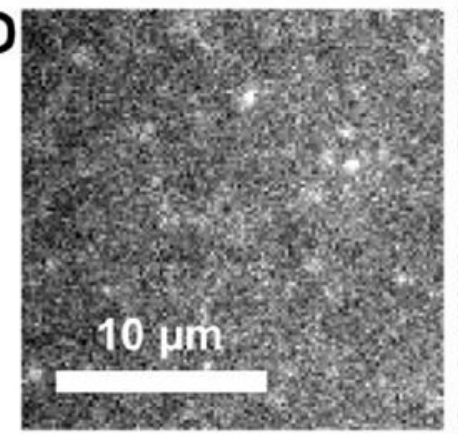

FtsZ $(2 \mu \mathrm{M})$ (35\% FtsZ-A488)

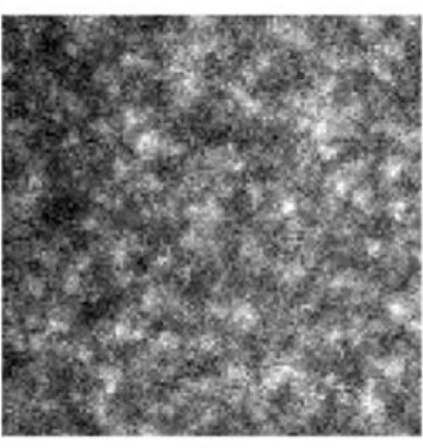

$>\quad F t s Z(2 \mu \mathrm{M})$

(35\% FtsZ-A488)

+ GTP (ii)
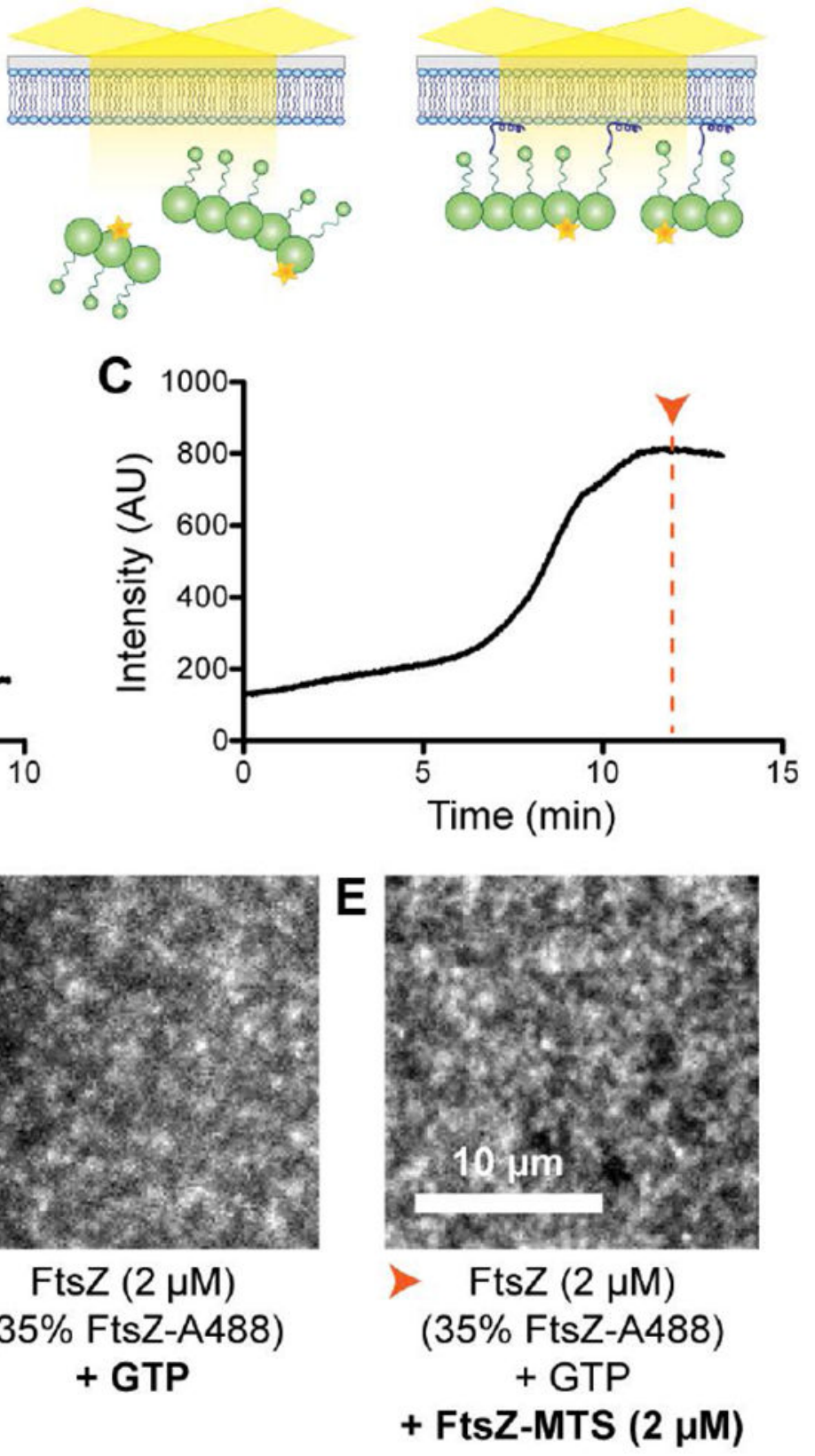

Figure 2.

FtsZ-MTS co-polymerizes with FtsZ and recruits protofilaments to SLBs. A. Schematic corresponding to the experimental setup in B - E. (i). Flow cell containing 20\% DOPG and $80 \%$ DOPC SLB equilibrated with $2 \mu \mathrm{M}$ FtsZ (35\% FtsZ-Alexa488), (ii) At steady state after flowing in $2 \mu \mathrm{M}$ FtsZ (35\% FtsZ-Alexa488) with GTP, and (iii) At steady state after subsequently flowing in $2 \mu \mathrm{M}$ FtsZ (35\% FtsZ-Alexa488) and $2 \mu \mathrm{M}$ FtsZ-MTS (unlabeled) with GTP. B. \& C. Fluorescence intensity on the SLB averaged over the frame $\left(\sim 400 \mu \mathrm{m}^{2}\right)$ over time. B. $2 \mu \mathrm{M}$ FtsZ (35\% FtsZ-Alexa488) with GTP was flowed at $0.5 \mu \mathrm{L}$ minute ${ }^{-1}$ into 
a flow cell equilibrated with $2 \mu \mathrm{M}$ FtsZ (35\% FtsZ-Alexa488). C. $2 \mu \mathrm{M}$ FtsZ (35\% FtsZAlexa488) and $2 \mu \mathrm{M}$ FtsZ-MTS with GTP was flowed at $0.5 \mu \mathrm{L}$ minute ${ }^{-1}$ into a flow cell equilibrated with $2 \mu \mathrm{M}$ FtsZ (35\% FtsZ-Alexa488) with GTP. D. Contrast enhanced TIRFM images showing structures corresponding to experiment in $\mathrm{B}$, immediately after beginning flow (blue arrowhead) and at steady state (green arrowhead). E. Contrast enhanced TIRFM image showing structures corresponding to experiment in $\mathrm{C}$ at steady state (orange arrowhead). Blue, green and orange arrowheads correspond to stages (i), (ii), and (iii) respectively as depicted in A. Scale bar $-10 \mu \mathrm{m}$. Reaction buffer contains $50 \mathrm{mM}$ HEPES pH 8.0, $0.1 \mathrm{mM}$ EDTA, $2.5 \mathrm{mM} \mathrm{MgCl} 2,300 \mathrm{mM} \mathrm{KCl}, 1 \%$ glycerol, $0.1 \mathrm{mg} \mathrm{mL}^{-1}$ casein (blocking agent), $0.5 \mathrm{mg} \mathrm{mL}^{-1}$ ascorbate. 

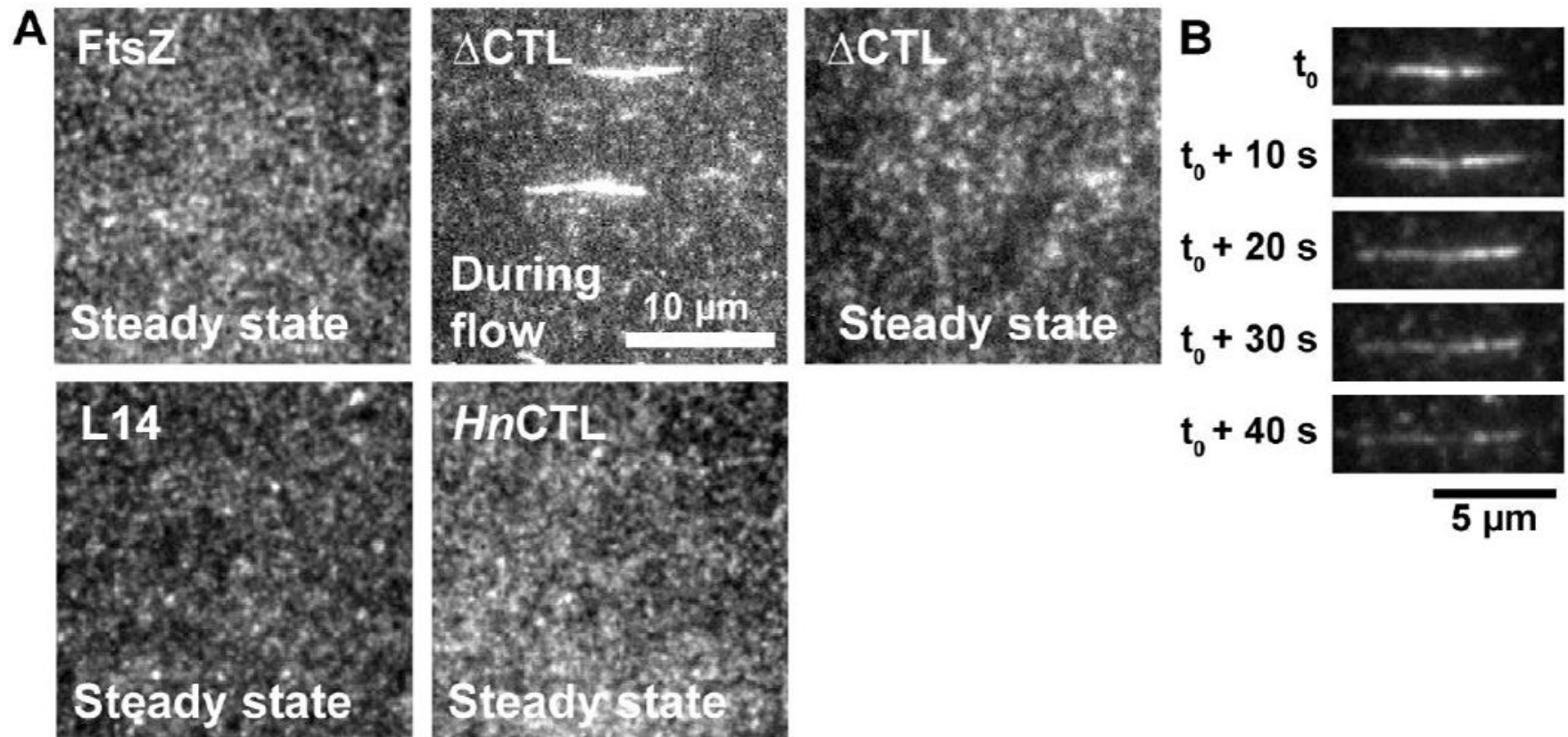

$t_{0}+40 s$

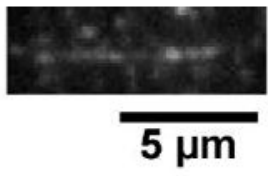

Figure 3.

$\triangle$ CTL protofilaments form extended bright structures in addition to dynamic clusters. A. Contrast enhanced TIRFM images of structures observed on 20\% DOPG 80\% DOPC SLBs for FtsZ or CTL variants flowed in with $2 \mathrm{mM}$ GTP at steady state or during flow. The FtsZ variants in each of the flow cells are $2 \mu \mathrm{M}$ FtsZ or CTL variant (6\% FtsZ-Alexa488 or corresponding Alexa488-labeled CTL variant) and $2 \mu \mathrm{M}$ C-terminal MTS fusions replacing the CTC of FtsZ or corresponding CTL variant. B. Representative contrast enhanced TIRFM images showing the disassembly of an extended bright structure formed by $\triangle \mathrm{CTL} / \Delta \mathrm{CTL}-$ MTS on the membrane over time. Scale bar $-10 \mu \mathrm{m}$. Reaction buffer contains $50 \mathrm{mM}$ HEPES pH 8.0, $0.1 \mathrm{mM}$ EDTA, $10 \mathrm{mM} \mathrm{MgCl} 2,300 \mathrm{mM} \mathrm{KCl}, 1 \%$ Glycerol, $0.1 \mathrm{mg} \mathrm{mL}^{-1}$ Casein (blocking agent), $0.5 \mathrm{mg} \mathrm{mL}^{-1}$ ascorbate. Structures presented are representative and were confirmed using at least 3 independent replicates. Extended bright structures were observed in all replicates for $\Delta \mathrm{CTL} / \Delta \mathrm{CTL}-\mathrm{MTS}$. 
A
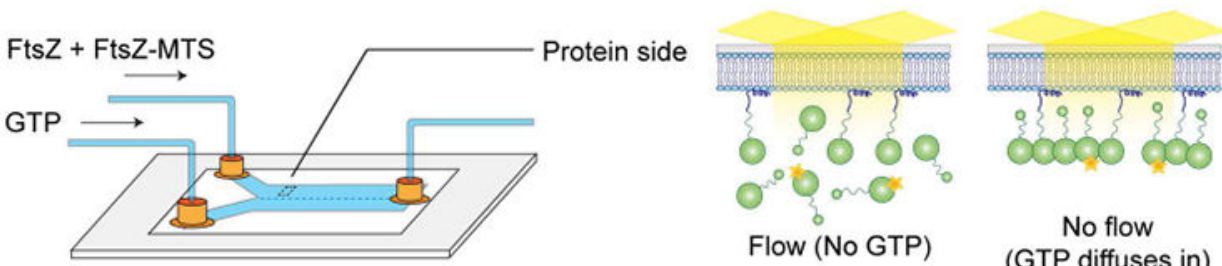

D

P diffuses in)

B

C

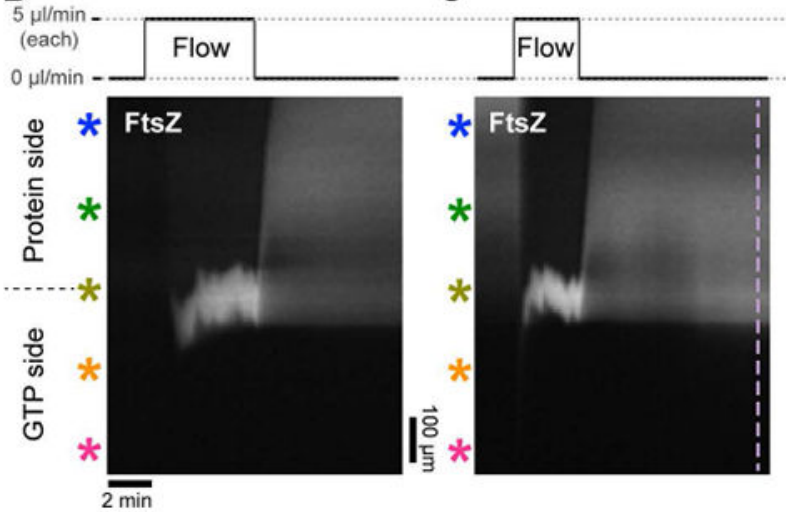

Flow

E

$\mathbf{F}$
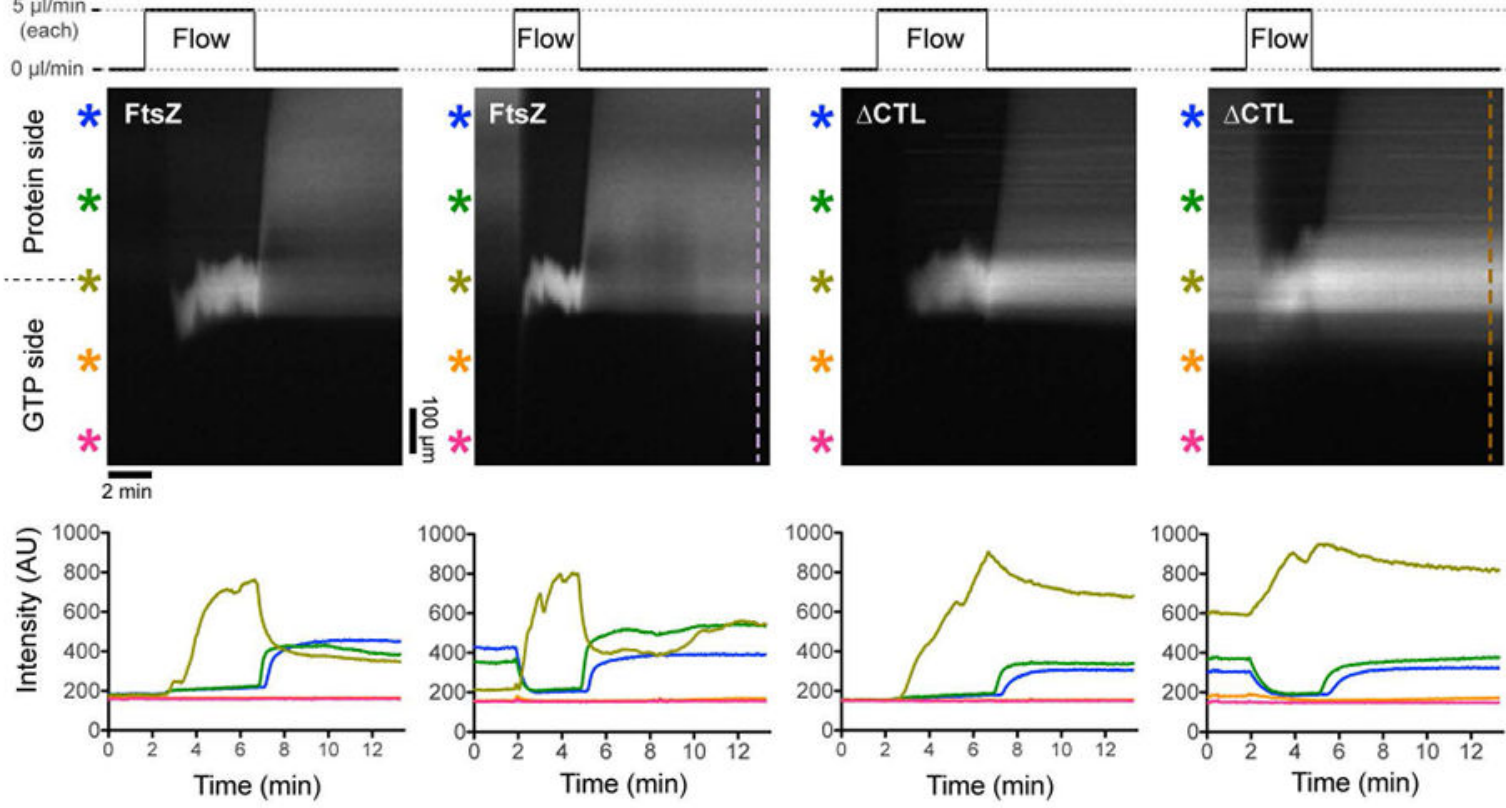

Figure 4.

FtsZ and $\triangle$ CTL polymers assemble distinct large-scale superstructures on SLBs. A.

Schematic depicting the two-inlet flow cell used for rapid initiation of polymerization and depolymerization. During flow, the protein channel side is depleted of GTP and FtsZ is predominantly monomeric. Immediately after flow is stopped, GTP diffuses into the protein channel side initiating FtsZ (6\% Alexa488 labeled) polymerization and recruitment to the membrane by copolymerizing with FtsZ-MTS, enabling visualization by TIRFM. B-E. Kymographs and corresponding fluorescence intensity vs time plots during periods of flow and no flow in the two-inlet flow cell. In the protein side, during flow, $2 \mu \mathrm{M} \mathrm{FtsZ} \mathrm{(6 \%}$ Alexa488 labeled) and $2 \mu \mathrm{M}$ FtsZ-MTS (unlabeled) $(\mathbf{B}, \mathbf{C})$ or $2 \mu \mathrm{M} \Delta \mathrm{CTL}$ (6\% Alexa488 labeled) and $2 \mu \mathrm{M} \Delta \mathrm{CTL}-\mathrm{MTS}$ (unlabeled) (D, E) is introduced at the flow rate of $5 \mu \mathrm{L}$ minute $^{-1}$. Simultaneously, in the GTP side, $2 \mathrm{mM}$ GTP is introduced at the same flow rate of $5 \mu \mathrm{L}$ minute ${ }^{-1}$. Time-lapse TIRF movies corresponding to the kymographs in B-E were obtained at 10× magnification. B. and D. represent kymographs and intensity plots corresponding to the first flow/stop cycle (flow up to $25 \mu \mathrm{L}$ at $5 \mu \mathrm{L}$ minute ${ }^{-1}$ for each channel into a fresh flow cell and then no flow to allow mixing), C. and E. correspond to subsequent flow-stop cycle (flow up to $15 \mu \mathrm{L}$ at $5 \mu \mathrm{L}$ minute ${ }^{-1}$ for each channel into the flow cell in B or D following steady state and then no flow to allow mixing). Scale bar $=100$ $\mu \mathrm{m}$ in spatial axis (vertical) and $2 \mathrm{~min}$ in temporal axis (horizontal) of kymograph, asterisks of different colors correspond to intensity plots of the same color denoting regions within the flow cell at varying distances perpendicular to the laminar boundary (and the direction of 
flow). F. Line plots along axis perpendicular to the direction of flow at steady state following re-initiation of assembly (after flow) at the indicated time points corresponding to kymographs in C. (FtsZ/FtsZ-MTS) and E. ( $\Delta$ CTL/ $\Delta$ CTL-MTS). Reaction buffer contains $50 \mathrm{mM}$ HEPES pH 8.0, $0.1 \mathrm{mM}$ EDTA, $10 \mathrm{mM} \mathrm{MgCl} 2,300 \mathrm{mM} \mathrm{KCl}, 1 \%$ glycerol, $0.1 \mathrm{mg}$ $\mathrm{mL}^{-1}$ casein (blocking agent), $0.5 \mathrm{mg} \mathrm{mL}^{-1}$ ascorbate. 

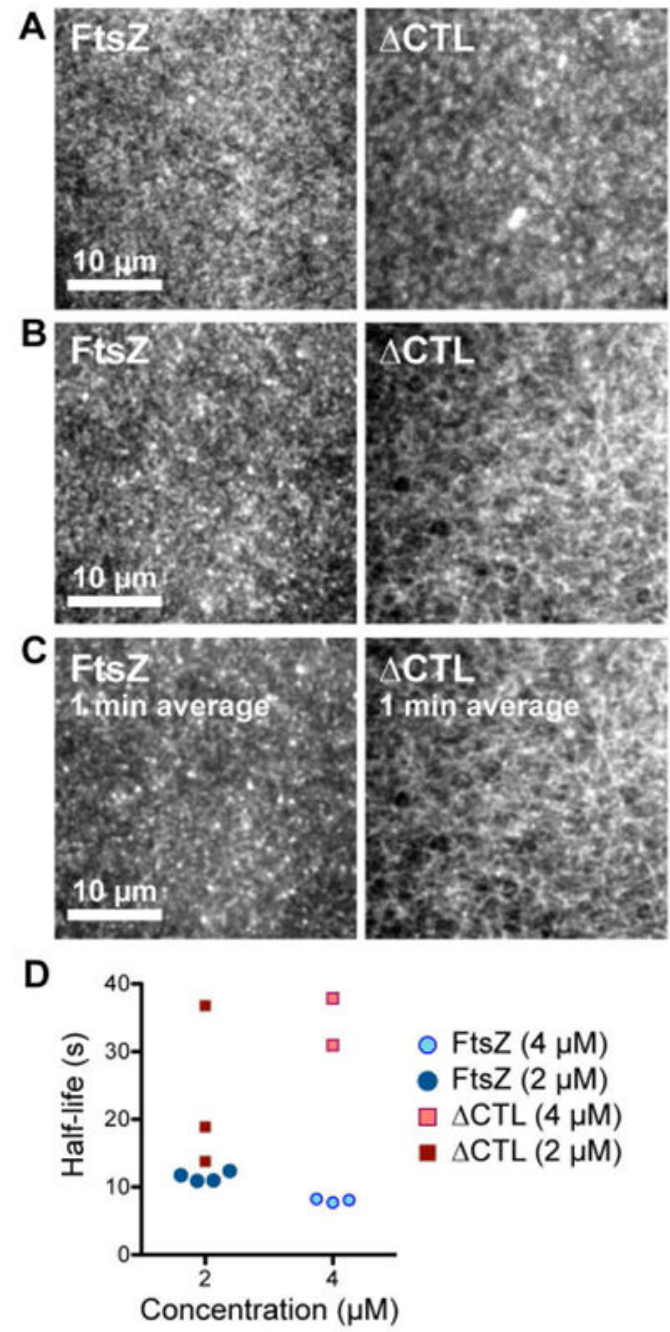

$$
\begin{aligned}
& \text { - FtsZ }(4 \mu \mathrm{M}) \\
& \text { - FtsZ }(2 \mu \mathrm{M})
\end{aligned}
$$$$
\square \mathrm{CTL}(4 \mu \mathrm{M})
$$$$
\text { - } \triangle \mathrm{CTL}(2 \mu \mathrm{M})
$$

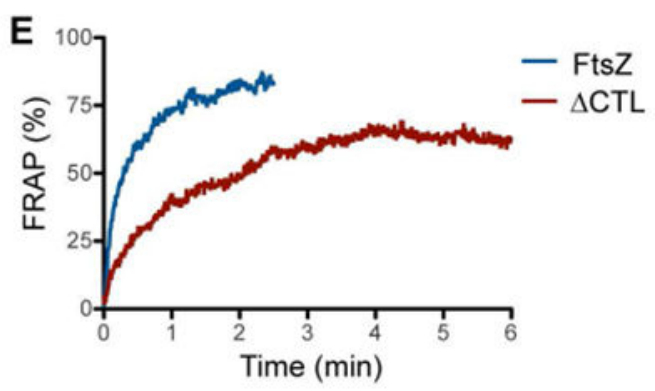

Figure 5.

$\triangle \mathrm{CTL}$ forms stable networks of straight filaments unlike WT FtsZ. A, B. Contrast enhanced micrographs of structures formed on SLBs at steady state after simultaneously flowing in 4 $\mu \mathrm{M}$ FtsZ (6\% Alexa488 labeled) and $4 \mu \mathrm{M}$ FtsZ-MTS (unlabeled) or $4 \mu \mathrm{M} \Delta \mathrm{CTL}$ (6\% Alexa488 labeled) and $4 \mu \mathrm{M} \Delta \mathrm{CTL}-\mathrm{MTS}$ (unlabeled) in the protein channel inlet and $4 \mathrm{mM}$ GTP in the GTP channel inlet and stopping flow. A. Structures formed farther from the original laminar boundary on the protein side. B. Structures formed closest to the original laminar boundary on the protein side. C. Time averages corresponding to the structures 
shown in B. obtained from taking averages over frames corresponding to a 1-minute time interval. Scale bar $-10 \mu \mathrm{m}$. D. Time until decrease in fluorescence intensity to halfmaximum value (half-life) for structures formed by FtsZ (6\% Alexa488 labeled) and FtsZMTS (unlabeled) or $\triangle \mathrm{CTL}$ (6\% Alexa488 labeled) and $\triangle \mathrm{CTL}-\mathrm{MTS}$ (unlabeled), following depletion of GTP. Half-life values were estimated from non-linear fits assuming one-phase exponential decay. E. Fluorescence recovery after photobleaching corresponding to structures showed in A (on the protein side, away from the original laminar boundary). Plot shows average of 3 replicates. Reaction buffer contains $50 \mathrm{mM}$ HEPES pH 8.0, $0.1 \mathrm{mM}$ EDTA, $10 \mathrm{mM} \mathrm{MgCl}_{2}, 300 \mathrm{mM} \mathrm{KCl}, 1 \%$ glycerol, $0.1 \mathrm{mg} \mathrm{mL}^{-1}$ casein (blocking agent), $0.5 \mathrm{mg} \mathrm{mL}^{-1}$ ascorbate. 